OPEN ACCESS

Edited by:

Xin Zhao,

McGill University, Canada

Reviewed by:

Florent Allais,

AgroParisTech Institut des Sciences et Industries du Vivant et de

L'environnement, France

Navnidhi Chhikara,

Guru Jambheshwar University of

Science and Technology, India

*Correspondence:

Io Antonopoulou

io.antonopoulou@/tu.se

Specialty section

This article was submitted to

Nutritional Immunology,

a section of the journal

Frontiers in Nutrition

Received: 15 September 2021 Accepted: 31 December 2021

Published: 07 February 2022

Citation:

Antonopoulou I, Sapountzaki E,

Rova $U$ and Christakopoulos $P$ (2022)

Ferulic Acid From Plant Biomass: A

Phytochemical With Promising

Antiviral Properties.

Front. Nutr. 8:777576.

doi: 10.3389/fnut.2021.777576

\section{Ferulic Acid From Plant Biomass: A Phytochemical With Promising Antiviral Properties}

\author{
lo Antonopoulou*, Eleftheria Sapountzaki, Ulrika Rova and Paul Christakopoulos \\ Biochemical Process Engineering, Division of Chemical Engineering, Department of Civil, Environmental and Natural \\ Resources Engineering, Luleå University of Technology, Luleå, Sweden
}

Plant biomass is a magnificent renewable resource for phytochemicals that carry bioactive properties. Ferulic acid (FA) is a hydroxycinnamic acid that is found widespread in plant cell walls, mainly esterified to polysaccharides. It is well known of its strong antioxidant activity, together with numerous properties, such as antimicrobial, anti-inflammatory and neuroprotective effects. This review article provides insights into the potential for valorization of FA as a potent antiviral agent. Its pharmacokinetic properties (absorption, metabolism, distribution and excretion) and the proposed mechanisms that are purported to provide antiviral activity are presented. Novel strategies on extraction and derivatization routes, for enhancing even further the antiviral activity of FA and potentially favor its metabolism, distribution and residence time in the human body, are discussed. These routes may lead to novel high-added value biorefinery pathways to utilize plant biomass toward the production of nutraceuticals as functional foods with attractive bioactive properties, such as enhancing immunity toward viral infections.

Keywords: antiviral activity, nutraceuticals, plant biomass, extraction, ferulic acid, metabolism, enzymatic derivatization, immunity

\section{INTRODUCTION}

The emerged global COVID-19 outbreak has led to significant efforts by the international research community for the development of novel vaccines, drugs and treatments to fight the pandemic. In parallel with vaccine development, already approved drugs and several natural bioactive compounds have been screened for their inhibitory activity against viruses in order to repurpose them for treatment $(1,2)$. It has been quite evident that better protection procedures, with high immune efficacy against viral infection, could play a significant role in future societal responses and preparedness to fight epidemics. In particular, the use of plant-derived nutraceuticals, not necessarily as drugs, but as food supplements with protective, curative and inactivating effects toward a variety of viral infections, may contribute to combat future pandemics or diseases that can affect not only a patient's heath but also their quality of life.

Ferulic acid (FA, 4-hydroxy-3-methoxycinnamic acid) is a promising phytochemical with strong antioxidant activity, whose antiviral potential has been underlined during the past years. It has a broad spectrum of other attractive biological properties including UV absorptive, skin whitening, antibacterial, anti-inflammatory, anti-thrombosis, neuroprotective and antitumor effects that highlights its importance for human health, nutrition and well-being $(3,4)$. Studies suggest that phenolics, such as FA, may have a vital role for combating human, animal or plant viruses. 
Additional to its low cytotoxicity and appreciable bioavailability score, FA is also a CYP1A2 inhibitor, which increases its drug half-life and averts serious drug interactions (5). CYP1A2 is a member of the cytochrome P450 mixed-function oxidase system and is involved in the metabolism of xenobiotics in the body involved in drug metabolism. Thus, FA could be a great candidate with good pharmacokinetic properties for control of viral infections.

In this review article, we will focus on the abundance, pharmacokinetics and proposed antiviral mechanisms of FA, while the potential of reported plant extracts containing FA as major metabolite and synthesized FA derivatives will be explored for their effect against a wide variety of human, animal and plant viruses. These valorization routes may lead to novel high-added value pathways to utilize plant biomass and respective residues toward the production of nutraceuticals with attractive bioactive properties.

\section{OCCURRENCE OF FA IN PLANT BIOMASS}

FA is the most abundant hydroxycinnamic acid found in plant biomass, followed to a lesser extent by $p$-coumaric acid (Figure 1A). It can be found in plant cells walls, mainly in its trans-form, usually esterified or etherified to the polymers within the lignocellulosic matrix. FA is commonly found in commelinid plants (rice, wheat, oats and pineapple), grasses, grains, vegetables, flowers, fruits, leaves, beans, seeds of coffee, artichoke, peanut and nuts (6). In graminaceous monocots, such as wheat and rice, FA can be found up to a $3 \%$ $\mathrm{w} / \mathrm{w}$ concentration and esterified to the $O-5$ hydroxyl group of FA and $\alpha$-L-arabinofuranose in glucuronoarabinoxylan (7) (Figure 1B). In few dicots, such as spinach or sugar beet, it is found at a concentration up to $1 \% \mathrm{w} / \mathrm{w}$ and esterified in pectin to the $O-2$ or $O-5$ hydroxyl group of $\alpha-\mathrm{L}$ arabinofuranose in arabinan or to the O-6 hydroxyl group of $\beta$-D-galactopyranose in (arabino-)galactan, both of which

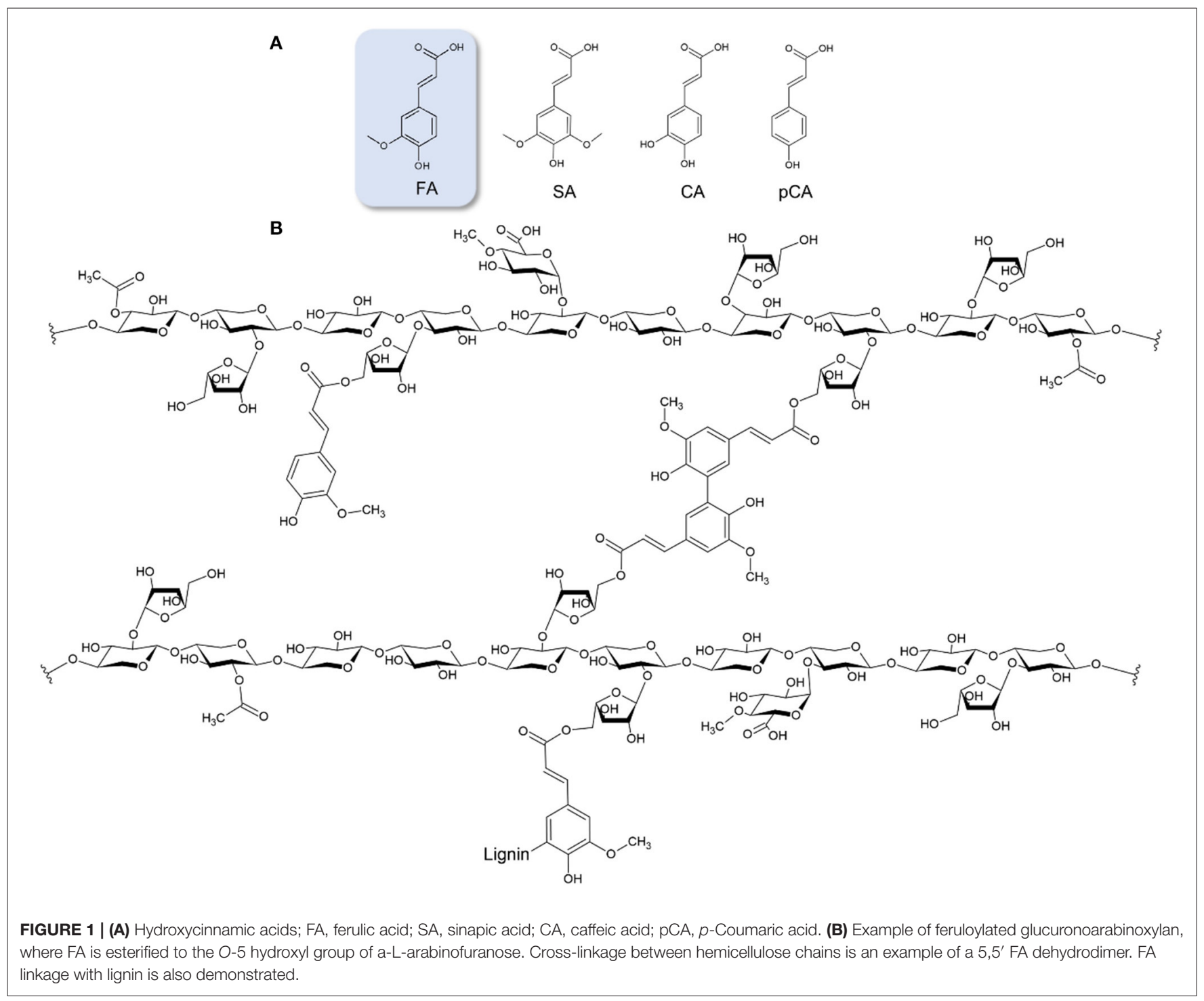


are side chains in rhamnogalacturonan I (8). FA has been found also esterified to the $O-4$ group of $\alpha$-D-xylopyranose in xyloglucans of bamboo (9). FA can be oxidatively cross-linked to form intermolecular ester bonds to another arabinoxylan and ether-ether bonds between hemicellulose and lignin. Different diferulic acids are detected; 8,5'-, 5,5'-, 8,4'-, 8, $8^{\prime}$ and less commonly $8,5^{\prime}$-(benzofuran)- and $8,8^{\prime}$-(aryl)-diFA (10), while FA trimers and tetramers have been also identified (11). Cross-linking of cell wall polysaccharides and lignin by hydroxycinnamic acids leads to a dramatic increase in mechanical strength, decelerates wall extension, and acts as barrier to block infection. FA is also detected in all families of gymnosperms, being ester-linked to the primary cell walls at a concentration between 0.01 and $0.16 \%$ w/w (12). In cereals, FA also exists in ester forms such as steryl ferulates ( $\gamma$-oryzanol), which was firstly recognized in 1954 in rice bran oil. Several methods have been recognized to extract FA by hydrolysis of $\gamma$-oryzanol (13).

\section{EXTRACTION AND PURIFICATION OF FA FROM PLANT BIOMASS}

To utilize FA for its bioactive properties, recovery in a form of extract or in a purified form is necessary (14). Extraction and further purification of FA and other $p$-hydroxycinnamic acids found in plant biomass can be done by different methods. Purification strategies can lead to obtaining the bioactive compound in pure form for direct use as bioactive agent or as a donor for further derivatization to synthesize novel agents with improved properties.

If FA is free esterified and not bound on the cell wall matrix, a direct extraction step can be applied in order to recover the valuable compound. Efficient extraction with green solvents has been reported, such aqueous ethanol solution (15) or hot water (16). Application of physical activation, such as ultrasound or microwave, may enhance extraction yields. If FA is encased in the cell wall matrix, pretreatment methods are usually applied, such as alkaline hydrolysis or enzymatic hydrolysis. Alkaline hydrolysis is a traditional procedure that is less green as it employs harsh chemicals and high temperatures, thus may have limitations for use in the cosmetic, pharmaceutical and food industries. Enzymatic hydrolysis of esterified FA can be done by the use of feruloyl esterases (EC 3.1.1.73), which consist accessory lignocellulose degrading enzymes (17-20). This class of enzymes, in nature, catalyzes the hydrolysis of the ester bond between FA and $\alpha$-L-arabinofuranose in hemicellulose, releasing FA and degrading the crosslinking between hemicellulose and lignin. As lignocellulose is highly recalcitrant and in order to improve accessibility of feruloyl esterases to release $p$ hydroxycinnamic acids, use of a consortium of cellulases and hemicellulases in often required. Use of enzymes has many advantages, such as high specificity leading to fewer degradation products, recyclability and preservation of the substrate, while the price of lignocellulose-degrading enzymatic cocktails has dropped significantly during the years making the process more affordable (21).
Hydrolysis methods often result in complex liquors due to the release of sugars, proteins, and other phenolic compounds. Thus, purification steps are needed to further increase extract purity. Purification of FA has been reported by ethanol washing $(22,23)$, adsorption on adsorbents, such as activated charcoal (22, 24), resins (25-30) and magnetic nanoparticles (31), membrane filtration (32-36) and chromatographic methods, such countercurrent high performance liquid chromatography (CCC) (37, 38). Examples on the aforementioned techniques are presented in Table 1.

\section{PHARMACOKINETICS OF FA: ABSORPTION, METABOLISM, DISTRIBUTION AND EXCRETION}

When ingested, FA can be absorbed in the stomach (40), small intestine; jejunum (41, 42) and ileum (42), and colon (43) (Figure 2). However, the major and most important site for FA absorption in the human body is the colon, where microbial esterases in the lumen of the intestine hydrolyze the esterified FA from the food matrix to generate free FA. Administration of FA sugar esters, such as 5-O-feruloyl-L-arabinofuranose or feruloylarabinoxylan, leads to the same metabolites being present in plasma as when free FA is administrated, reinforcing the belief that FA esters are broken down to FA and then follow the same metabolic route (44). However, there is also a hypothesis supporting that FA sugar esters are absorbed intact and then hydrolyzed in mucosal cells or in circulation. In any case, research shows that free FA can be absorbed before reaching the ileum, but when bound to fiber, it has to be degraded in the hindgut in order to be absorbed and further metabolized (45).

Studies on an in vitro model for human colonic epithelium showed that FA is mainly transported in its free form, after released from its ester form by esterases. FA is suggested to be absorbed by a combination of mechanisms: passive transcellular diffusion and facilitated transport. Transcellular diffusion is dependent on the $\mathrm{pH}$ of the environment, the $\mathrm{pKa}$ and the hydrophobicity of the compound. FA, with a pKa equal to 4, could be transported in an undissociated form via a passive diffusion mechanism in the low gastric $\mathrm{pH}$ environment (46). However, this might not be the case for intestinal absorption, as FA would not maintain its undissociated form in the weak acidic environment of the intestine $(\mathrm{pH}>5)$ (47).

Studies regarding FA absorption in rats through the jejunal mucosa have indicated a potential mechanism involving a $\mathrm{Na}(+)$ dependent transport mechanism (41), whereas Itagaki et al. (48) also mention the possibility of a $\mathrm{H}+$-dependent carrier, testing the uptake of FA in Caco- 2 cells. However, such transporters have not been identified yet. According to Konishi and Shimizu (49), transepithelial transport of FA across Caco-2 cell monolayers was suggested to be related to binding to a monocarboxylic acid transporter (MCT), in particular to MCT1. However, in one of their more recent studies it is eventually supported that FA transport is achieved through another MCT type transporter and not MCT1 (50). MCTs are believed to be involved in gastric absorption, apart from the colonic, but the specific type of 
TABLE 1 | Examples of reported methods on the pretreatment of plant biomass and further purification of FA.

\begin{tabular}{|c|c|c|c|c|}
\hline Plant source & Pretreatment step & Purification step & Yield/Purity/Efficiency & References \\
\hline Wheat bran & $\begin{array}{l}\text { Defatted with hexane, alkaline } \\
\text { hydrolysis, acidification to } \mathrm{pH} 2 \text {, } \\
\text { extraction with ethyl ester (three } \\
\text { times) }\end{array}$ & $\begin{array}{l}\text { Counter-current chromatography followed } \\
\text { by pH-zone-refining counter-current } \\
\text { chromatography }\end{array}$ & 98-99\% purity & (38) \\
\hline Brewer's spent grain & $\begin{array}{l}\text { Dilute sulfuric acid hydrolysis } \\
\text { followed by alkaline hydrolysis }\end{array}$ & Ethanol extraction & $46.17 \mathrm{mg} / 100 \mathrm{~g} \mathrm{BSG}$ & (23) \\
\hline Sugar cane bagasse & Alkaline hydrolysis & $\begin{array}{l}\text { Adsorption by powdered activated } \\
\text { charcoal and further treatment by anion } \\
\text { macroporous resin exchange } \\
\text { chromatography }\end{array}$ & $\begin{array}{l}\text { FA could be totally washed out by } 0.2 \\
\mathrm{~mol} / \mathrm{L} \mathrm{NaOH} \text {, high purity obtained }\end{array}$ & $(24)$ \\
\hline Radix Angelicae Sinensis & $\begin{array}{l}\text { Microwave-assisted ethanol } \\
\text { extraction }\end{array}$ & $\begin{array}{l}\text { High-speed counter-current } \\
\text { chromatography }\end{array}$ & $>98 \%$ purity & $(37)$ \\
\hline Wheat bran & Enzymatic hydrolysis & $\begin{array}{l}\text { Nanofiltration membranes (MWCO } \\
\text { between } 600 \text { and } 800 \mathrm{~g} \mathrm{~mol}^{-1} \text { ) }\end{array}$ & $85 \%$ retention for FA & (34) \\
\hline Destarched wheat bran & Alkaline hydrolysis & Adsorption on Amberlite XAD4 resin & $\begin{array}{l}49.9 \mathrm{mg} \mathrm{FA} / \mathrm{g} \text { resin, } 99.5 \% \text { recovery using } \\
\text { methanol as eluent }\end{array}$ & $(27)$ \\
\hline Wheat bran & $\begin{array}{l}\text { Ultrasound-assisted ethanol } \\
\text { extraction }\end{array}$ & Adsorption on magnetic nanoparticles & 77.9-97.5\% recovery & $(31)$ \\
\hline Corn fiber & Alkaline hydrolysis & Ultrafiltration membrane (Nadir UP 150) & $98.0 \%$ rejection of arabinoxylans & (35) \\
\hline Corn bran & Alkaline hydrolysis & Adsorption on mesoporous carbon & $\begin{array}{l}\text { Much higher adsorption capacity for FA } \\
\text { than those of activated charcoal, other } \\
\text { activated carbon materials, and } \\
\text { macroporous resins }\end{array}$ & (39) \\
\hline $\begin{array}{l}\text { Sugar beet pulp, maize } \\
\text { bran, wheat bran, rice bran }\end{array}$ & Enzymatic hydrolysis & Adsorption on Amberlite XAD-4 resin & $1.9 / 5.5 \mathrm{mg} F A / g$ & (29) \\
\hline Brewer's spent grain & Alkaline hydrolysis & $\begin{array}{l}\text { Adsorption by synthetic resin (Lewatit } \\
\text { VPOC1064 MD PH®) }\end{array}$ & Partial purification & $(28)$ \\
\hline Wheat bran & Enzymatic hydrolysis & $\begin{array}{l}\text { Demineralization by homopolar } \\
\text { electrodialysis, adsorption on weak } \\
\text { anion-exchange resin (Amberlyst A21- } \\
\text { Dow) }\end{array}$ & $\begin{array}{l}52 \% \text { of FA was released by enzymatic } \\
\text { hydrolysis, of which } 83 \% \text { was crystallized } \\
\text { with purity } 90-95 \%\end{array}$ & $(26)$ \\
\hline $\begin{array}{l}\text { Flax shives, wheat bran and } \\
\text { corn }\end{array}$ & Alkaline hydrolysis, pressurized & $\begin{array}{l}\text { Ultrafiltration and ethanol } \\
\text { solubilization/centrifugation cycles ( } 2 \\
\text { times) }\end{array}$ & $56 \%$ efficiency, clean TLC and FTIR (FA) & $(32)$ \\
\hline Corn bran & Alkaline hydrolysis & $\begin{array}{l}\text { Ultrafiltration (5,000 Da MWCO), } \\
\text { nanofiltration }\end{array}$ & $\begin{array}{l}91.8 \% \text { recovery, } 8.47 \mathrm{~g} \text { FA crystals } / \mathrm{kg} \\
\text { corn bran with } 84.45 \% \text { purity }\end{array}$ & (36) \\
\hline \multirow[t]{3}{*}{ Grass } & $\begin{array}{l}\text { Dilute sulfuric acid followed by } \\
\text { alkaline hydrolysis }\end{array}$ & Ethanol extraction & $30.9 \mathrm{~g} \mathrm{FA} / 100 \mathrm{~g}$ grass & $(22)$ \\
\hline & & $\begin{array}{l}\text { Adsorption with activated charcoal or } \\
\text { polyvinylpolypyrrolidone (PVPP) }\end{array}$ & $3.3 \mathrm{~g} \mathrm{FA} / 100 \mathrm{~g}$ grass & \\
\hline & & & $0.5 \mathrm{~g} \mathrm{FA} / 100 \mathrm{~g}$ grass & \\
\hline Sugar beet pulp & $\begin{array}{l}\text { Twin-screw extraction and } \\
\text { enzymatic hydrolysis }\end{array}$ & $\begin{array}{l}\text { Microfiltration }(3 \mu \mathrm{m}) \text {, adsorption on } \\
\text { activated charcoal }\end{array}$ & $\begin{array}{l}50 \% \text { purity (FA), } 6 \text { cycles to achieve } 97 \% \\
\text { efficiency }\end{array}$ & (33) \\
\hline Wheat bran & Enzymatic hydrolysis & Adsorption on weak anionic resin & $15 \mathrm{~g} / \mathrm{L}, 67 \%$ recovery & $(25)$ \\
\hline
\end{tabular}

transporter has not been determined (51). Both of the previously mentioned mechanisms give free FA on the serosal side, with only a small number of conjugated forms.

FA is further metabolized mainly by the liver, where the major metabolites are FA-conjugated forms. The major metabolite of FA is FA-sulfoglucuronide, with other common conjugated forms being FA-glucuronide and FA-sulfate (45). These kind of conjugations is a way of detoxifying the compound and increasing its polarity (52). Additional FA metabolites include $m$ hydrophenylpropionic acid, feruloylglycine, dihydroferulic acid, vanillic acid, vaniloylglycine, feruloyl-sulfate and trans-feruloyl4 - $O$ - $\beta$-D-glucuronide $(43,53)$. The conjugation reaction for the formation of FA-sulfoglucuronide, FA-glucuronide and FAsulfate is carried out by the activities of sulfotransferases and UDP glucuronosyl transferases, while the intestinal mucosa and kidney may take part in this process. Conjugation of FA may be dose dependent as very high doses of FA may saturate the conjugation enzymes, leading to accumulation of free FA in the plasma. Apart from conjugation, metabolic reactions include reduction of the double bond, demethylation, dehydroxylation of 


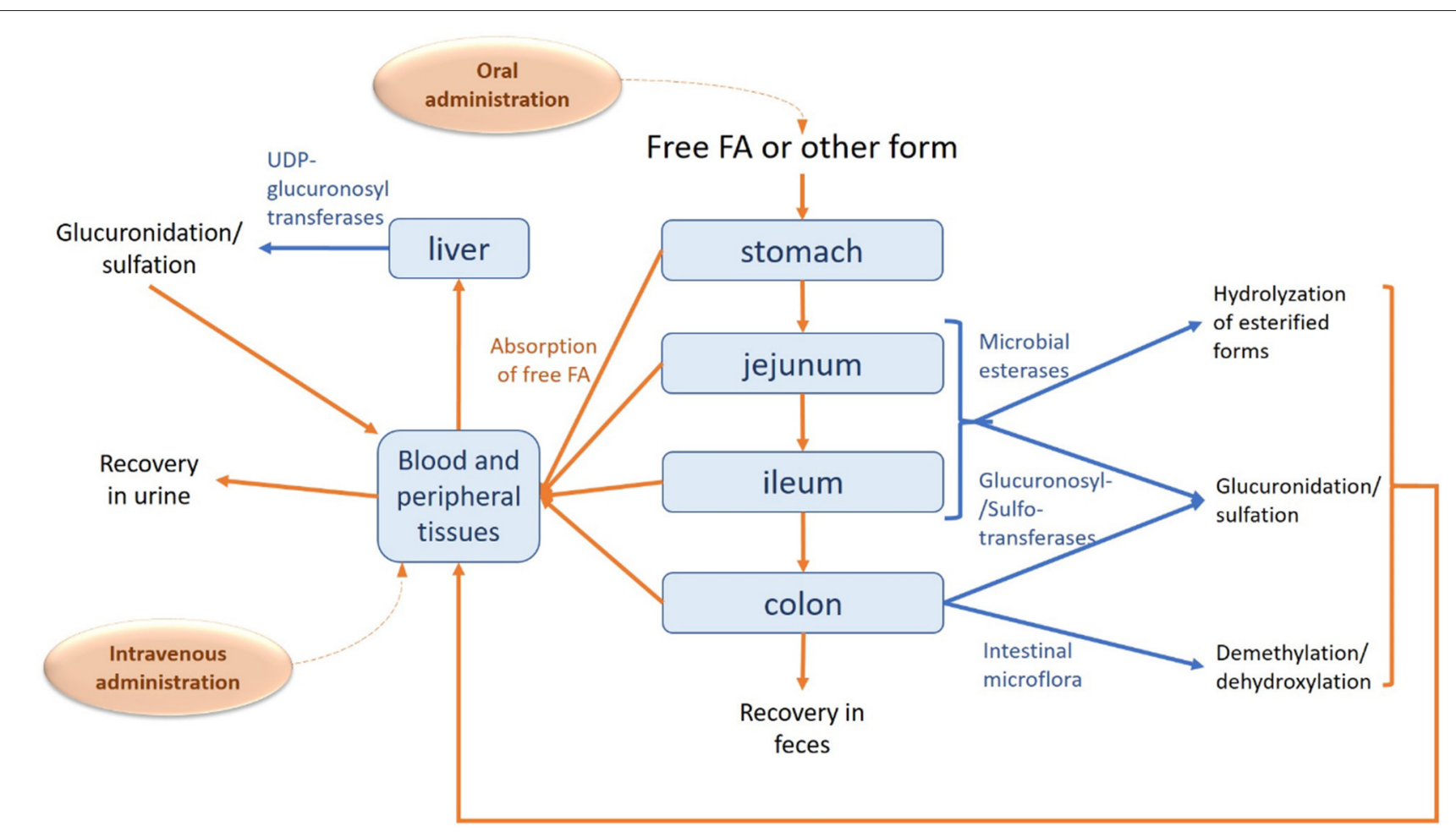

FIGURE 2 | Absorption, metabolism and excretion of FA in human body. The route of FA is described with orange arrows, while the metabolic modifications are shown in blue.

C3 or C4 $(54,55)$, as well as methylation $(53,56)$. The efflux of the conjugates is also transporter(s)-dependent (57).

Maximum plasma concentration as investigated in humans is reached $24 \mathrm{~min}$ after ingestion (57), whereas Li et al. (58) report a much lower value $(2 \mathrm{~min})$ in rats. Half-lives for the distribution and elimination phases in the latter case have been calculated at $\sim 10$ and $60-106 \mathrm{~min}$, respectively. It is suggested that the amount of FA that does not proceed to the liver, $~ 50 \%$ of the initial intake, is found in the bloodstream, gastric mucosa and peripheral tissue (59). Another study reports that from an initial intake of FA $(521 \mu \mathrm{mol} / \mathrm{kg}), 14 \mu \mathrm{g} / \mathrm{g}$ was detected in the heart, $82 \mu \mathrm{g} / \mathrm{g}$ in the kidney, $22 \mu \mathrm{g} / \mathrm{g}$ in the spleen, $34 \mu \mathrm{g} / \mathrm{g}$ in the lung, $28 \mu \mathrm{g} / \mathrm{g}$ in the liver, $15 \mu \mathrm{g} / \mathrm{g}$ in the uterus and $2.6 \mu \mathrm{g} / \mathrm{g}$ in the brain of rats, $30 \mathrm{~min}$ post-administration (60). The main distributor of FA in the body seems to be serum albumin (61). The source, form, and amount of FA ingested influences the ratio of the different forms available in blood for further uptake by the tissues. According to a different study, total plasma FA concentration in rats included no more than $24 \%$ free FA, and $76 \%$ of glucuronidated and sulfoglucuronidated forms which peaked $30 \mathrm{~min}$ after oral administration (52).

In humans, excretion of FA via urine reaches a plateau after $\sim 7-9 \mathrm{~h}$ after ingestion (62). The recovery of free FA in urine was reported to be around 5\% in both rats and humans (63), whereas the excretion of total FA, both in its free form as well as in its conjugated forms, mostly as feruloyl glucuronide, reaches 11$25 \%$ (62). The percentage of the conjugated, and more specifically sulfoglucuronidated FA, compared to the total excreted amount is even higher in urine than in plasma, reaching $84 \%$ (52). Only $0.5-0.8 \%$ of ingested FA has been found in feces of rats, indicating very efficient absorption rate for FA $(64,65)$.

Zhao et al. (40) suggest that oral administration of free FA results in a quick absorption, but also quick excretion of FA, whereas administration through a capsule would contribute to maintaining the desirable concentration of FA in the body for a longer period of time. For instance, a four times enhancement of the bioavailability of encapsulated FA compared to free form has been observed in rats (66). Ou and Kwok (67) report that the most effective way for FA to enter enterohepatic circulation, as tested in rats, is to be ingested in its natural form, as part of plant tissue. However, Adam et al. (65) mention that the recovery of FA is low when it has been consumed in the form of whole cereal, indicating that absorption is higher when FA is in its free form. The bioavailability of FA is affected by the complexity of the polysaccharide matrix to which FA is bound. For example, the presence of diferulic acids is concomitant to cross-links between polysaccharide chains that impede hydrolyzation by the responsible enzymes (68). On the contrary, Rondini et al. (69) observed that urinary excretion of FA after bran consumption was 15 -fold less than that after intake of free FA. Such differences of urinary excretion of FA were still observed with a perfusion of rat intestine with pure $\mathrm{FA}$, allowing the recovery of $50 \%$ of the intake dose and only $3.2 \%$ after bran consumption. Moreover, wheat arabinoxylan, that is rich in esterified FA, has been shown 
to act as a prebiotic, stimulating growth of good bacteria such as lactobacilli and bifidobacteria in the human gastrointestinal tract $(70,71)$. Studies on metabolism, distribution and excretion of free FA or derivatives after intravenous administration have not been yet reported.

\section{PROPOSED MECHANISMS FOR ANTIVIRAL ACTIVITY}

During the past years, FA has been suggested to play an important protective role against viruses. There are different types of mechanisms responsible for this effect. FA is known to promote induction of heme oxygenase-1 (HO-1) and hence may show utility for boosting type 1 interferon response (IFNs) that regulates the activity of the immune system $(72,73)$. Sodium ferulate was found to activate toll-like receptors (TRL) 7 and 9 in influenza A-infected mice, which are proteins that recognize viral nucleic acids and rapidly trigger different signaling cascades that contribute to the production of IFNs to antiviral defense. This effect could be secondary to $\mathrm{HO}-1$ induction, and possibly reflects an additional beneficial effect of FA (74).

The anti-inflammatory impact of nutraceuticals, such as FA, might also be expected to quell the excessive inflammatory reaction within lung parenchyma evoked by viral infections, whose lethality is mediated by an acute respiratory distress syndrome. In particular, FA has been reported to hinder the production of inflammatory proteins induced by viral infections, such as macrophage inflammatory protein-2 (MIP-2) in respiratory syncytial virus (RSV)-infected cells (75) and IL-8 in murine macrophage cells infected by influenza (76). Thus, FA could be a natural solution for decreasing such response both by suppressing viral spread, and by dampening pro-inflammatory signaling in endothelial cells that in turn promotes influx of inflammatory cells. Mccarty and Dinicolantonio (77) suggested that a very low daily dosage ranging from 500 to $1,000 \mathrm{mg}$ of FA could aid the control of influenza and corona viruses.

The most important and major impact of FA is its potential to have an inhibiting effect against key viral proteins that are associated with viral functions, such as replication. Two analogs of FA have been proposed to have inhibiting effects to neuraminidase, a surface glycoprotein vital for the viral replication and an important target for antiinfluenza drug, using molecular docking (78). Virtual screening of 300 immunomodulatory medicinal compounds revealed that FA, together with only five other natural phenolic compounds (arzanol, genistein, resveratrol, rosmanol and thymohydroquinone), had significant interaction with the SARS CoV-2 viral proteins (SARS coronavirus peptidase (2GTB), severe acute respiratory syndrome coronavirus 2 (SARS CoV2) protease 3CL (Mpro) (6LU7), spike glycoprotein (6VSB), NSP-9 replicase protein (6W4B) and NSP-15 endoribonuclease (6VWW) by forming hydrogen bonds with the active site residues with low binding energy, inhibiting their activity (79). A study screening 548 anti-viral natural and synthetic antiviral compounds in silico also showed that FA together with caffeic acid were found to inhibit a $3 \mathrm{D}$ model of SARS
CoV-2 membrane protein (80). These studies underline the potential important function of $\mathrm{FA}$ as a natural inhibitor of SARS-CoV-2.

Moreover, FA has been found to inhibit viral replication by hindering porcine parvovirus (PPV)-induced intrinsic PK-15 cell apoptosis by suppressing the expression of proteins that play a role in the cell apoptosis pathway (Cyt-c, Apaf-1, Caspase-9) and $\mathrm{Bcl}-2$ group proteins in particular. In addition, FA prevents generation of reactive oxygen species (ROS) by blocking caspase activity (81).

\section{ANTIVIRAL ACTIVITY OF PLANT EXTRACTS CONTAINING FA}

Plant extracts have been long known in folk medicine for their therapeutic properties. During the past decades, a variety of extracts rich in bioactive phenolic compounds, where FA is found as main or secondary component, have been evaluated for their activity against viruses. Except for their antiviral effect, these extracts demonstrate a rich profile of attractive bioactive properties, including antioxidant, anti-inflammatory and antiproliferative activities, and could be a valuable asset for boosting our immunity against various conditions, including viral infection. Plant extracts containing FA and evaluated for their antiviral activity come from angiosperms, in particular herbaceous plants with no woody stem above ground, and various flowering plants including shrubs and trees. An overview of the assessed plant extracts is presented in Table 2.

Lemon balm (Melissa officinalis L.; Lamiaceae) is a perennial herbaceous plant of the mint family that has been used in traditional medicine for nervous complaints, lower abdominal disorders and for treating Herpes lesions. Among lemon balm leaf extracts containing FA, caffeic acid and rosmarinic acid as key extractives, highest virucidal effect against simple herpex virus type 1 (HSV-1) was observed for the aqueous extract, where the infection activity was repressed completely at $6 \mathrm{~h}$ after treatment at maximum tolerable concentration in infected rabbit kidney (RK) cells (82). More recently, the inhibitory effect of lemon balm hydroalcoholic extract has been demonstrated for HSV type 2 (HSV-2) replication by the cytopathic effect inhibition assay on Vero cells (100) and for adenovirus (ADV) evaluated on HEp2 cells using the MTT (3-[4,5-dimethylthiazol-2-yl]-2,5diphenyltetrazolium bromide) assay (101).

FA, as a main active constituent of Cimicifuga heracleifolia extract, along with isoferulic acid, has also been reported as active against respiratory syncytial virus (RSV) by reducing the virus-induced production of inflammatory proteins MIP-2. The levels of MIP-2 observed on infected murine macrophage cells were reduced to 74.4 and $42.8 \%$ of the control at pure FA concentrations of 50 and $500 \mu \mathrm{g} / \mathrm{ml}$ respectively, whereas isoferulic acid reduced levels to 58.0 and $35.6 \%$ of the control at the respective concentrations (75). Cimicifuga rhizoma methanol extract has also been found to suppress viral replication of mouse hepatitis virus (MHV-A59), with a half maximal effective concentration $\left(E_{50}\right)$ of $19.4 \mu \mathrm{g} / \mathrm{ml}$ calculated by the plaque assay (102). The rhizoma of Cimicifuga species (bugbane; 
TABLE 2 | Antiviral effect of plant extracts containing FA.

\begin{tabular}{|c|c|c|c|c|c|c|}
\hline Plant source & Extraction method ${ }^{a}$ & $\begin{array}{l}\text { Major detected } \\
\text { compounds }\end{array}$ & Virus & Antiviral effect & Cytotoxicity & References \\
\hline $\begin{array}{l}\text { Melissa officinalis } \mathrm{L} \text {. } \\
\text { (lemon balm) }\end{array}$ & $\begin{array}{l}\text { Aqueous extraction of leaves, filtration } \\
\text { and spray drying }\end{array}$ & $\begin{array}{l}\text { FA, caffeic acid, } \\
\text { rosmarinic acid }\end{array}$ & HSV-1 & $\begin{array}{l}\text { Complete viral } \\
\text { inhibition after } 6 \mathrm{~h} \text { at } \\
\text { maximum tolerable } \\
\text { concentration of the } \\
\text { extract }\end{array}$ & $\begin{array}{l}\text { Maximum tolerable } \\
\text { concentration: } \\
0.25 \%\end{array}$ & (82) \\
\hline $\begin{array}{l}\text { Cimicifuga } \\
\text { heracleifolia } \\
\text { (bugbane) }\end{array}$ & - & $\begin{array}{l}\text { FA, isoferulic acid } \\
\text { (pure compounds) }\end{array}$ & RSV & $\begin{array}{l}42.8 \% \text { lower MIP-2 } \\
\text { levels compared to } \\
\text { the control at } 500 \\
\mu \mathrm{M} \text { FA }\end{array}$ & $\begin{array}{l}\text { Cells }>95 \% \text { viable } \\
\text { when treated with } \\
500 \mu \mathrm{M} \text { compound }\end{array}$ & (75) \\
\hline \multirow[t]{3}{*}{$\begin{array}{l}\text { Plantago major } \mathrm{L} \text {. } \\
\text { (broadleaf plantain) }\end{array}$} & $\begin{array}{l}\text { Successive hot water extraction of } \\
\text { whole plant, filtration, concentration in } \\
\text { vacuo and lyophilization }\end{array}$ & $\begin{array}{l}\text { Baicalein, baicalin, } \\
\text { luteolin, aucubin, } \\
\text { caffeic acid, } \\
\text { chlorogenic acid, } \\
\text { FA, } p \text {-coumaric acid, } \\
\text { oleanolic acid, } \\
\text { ursolic acid, vanillic } \\
\text { acid (based on } \\
\text { literature) }\end{array}$ & HSV-2 & $\mathrm{EC}_{50}=843 \mu \mathrm{g} / \mathrm{ml}$ & $\mathrm{CC}_{50}=1,809 \mu \mathrm{g} / \mathrm{ml}$ & (83) \\
\hline & - & FA (pure compound) & ADV-8 & $\mathrm{EC}_{50}=52.5 \mu \mathrm{g} / \mathrm{ml}$ & $\mathrm{CC}_{50}=92.6 \mu \mathrm{g} / \mathrm{ml}$ & \\
\hline & & & ADV-11 & $\mathrm{EC}_{50}=23.3 . \mu \mathrm{g} / \mathrm{ml}$ & & \\
\hline Ficus carica (fig) & $\begin{array}{l}\text { Successive methanol extraction of the } \\
\text { plant latex, filtration and evaporation, } \\
\text { followed by silica gel chromatography } \\
\text { eluting with hexane and after through } \\
\text { hexane-ethyl acetate, drying under } \\
\text { reduced pressure }\end{array}$ & $\begin{array}{l}\text { Caffeic acid, } \\
\text { 3,4-dihydrobenzoic } \\
\text { acid, } \\
\text { p-OH-phenylacetic } \\
\text { acid, p-coumaric } \\
\text { acid, luteolin, } \\
\mathrm{N} \text {-argenine, FA } \\
\text { (majority in the } \\
\text { extract) }\end{array}$ & $\begin{array}{l}\text { HSV-1 } \\
\text { ECV-11 }\end{array}$ & $\begin{array}{l}100 \% \text { blocked } \\
\text { replication of the } \\
\text { virus and virucidal } \\
\text { activity when } \\
\text { incubated with the } \\
\text { extract for } 1 \mathrm{~h} \text { with } \\
78 \mu \mathrm{g} / \mathrm{ml} \text { extract }\end{array}$ & $\begin{array}{l}\text { No cytotoxic effect } \\
\text { at tested } \\
\text { concentrations }\end{array}$ & (84) \\
\hline \multirow[t]{4}{*}{ Kalanchoe gracilis } & $\begin{array}{l}\text { Cold-pressing of leaves, filtration and } \\
\text { freeze drying }\end{array}$ & $\begin{array}{l}7.59 \% \text { w/w FA, } \\
0.33 \% \mathrm{w} / \mathrm{w}, \\
\text { quercetin, } 0.16 \% \\
\text { w/w kaempferol }\end{array}$ & EV71 & $\begin{array}{l}\mathrm{I}_{50}=35.88 \mu \mathrm{g} / \mathrm{ml} \\
\text { (plaque reduction } \\
\text { assay on RD cells) }\end{array}$ & $\begin{array}{l}\text { No cytotoxicity at a } \\
\text { range of } \\
1-500 \mu \mathrm{g} / \mathrm{ml}\end{array}$ & (85) \\
\hline & & & & $\begin{array}{l}\mathrm{IC}_{50}=40.82 \mu \mathrm{g} / \mathrm{ml} \\
\text { (cell-based FRET } \\
\text { assay) }\end{array}$ & & \\
\hline & & & CVA16 & $\begin{array}{l}\mathrm{I}_{50}=42.91 \mu \mathrm{g} / \mathrm{ml} \\
\text { (plaque reduction } \\
\text { assay on RD cells) }\end{array}$ & & \\
\hline & & & & $\begin{array}{l}\mathrm{I}_{50}=47.87 \mu \mathrm{g} / \mathrm{ml} \\
\text { (cell-based FRET } \\
\text { assay) }\end{array}$ & & \\
\hline
\end{tabular}


TABLE 2 | Continued

\begin{tabular}{|c|c|c|c|c|c|c|c|}
\hline Plant source & Extraction method ${ }^{a}$ & & $\begin{array}{l}\text { Major detected } \\
\text { compounds }\end{array}$ & Virus & Antiviral effect & Cytotoxicity & References \\
\hline $\begin{array}{l}\text { Eugenia } \\
\text { singampattiana }\end{array}$ & $\begin{array}{l}\text { Extraction of dried leaves with } \\
\text { methanol or water, centrifugation and } \\
\text { evaporation-redissolution with } \\
\text { distilled water }\end{array}$ & & $\begin{array}{l}\text { 4-hydroxybenzoic } \\
\text { acid, caffeic acid, } \\
\text { rutin, FA, coumaric } \\
\text { acid } \\
\text { epigallocatechin } \\
\text { gallate, quercetin, } \\
\text { myricetin, } \\
\text { kaempferol }\end{array}$ & PRRSV & $\begin{array}{l}\text { Inhibition observed } \\
\text { with } 25 \mu \mathrm{g} \text { of } \\
\text { methanol extract or } \\
75-100 \mu \mathrm{g} \text { of water } \\
\text { extract }\end{array}$ & - & (86) \\
\hline \multirow[t]{6}{*}{ Propolis } & $\begin{array}{l}\text { Propolis Extract } \mathrm{ACF} \otimes \text { (specific } \\
\text { purified ethanol extract) }\end{array}$ & & $\begin{array}{l}\text { Aromatic acids incl., } \\
\text { E-p-coumaric acid } \\
\text { (main), benzoic acid, } \\
\text { FA ( 3\%), fatty } \\
\text { acids, esters incl. } \\
\text { benzyl p-coumarate, } \\
\text { flavonoids, } \\
\text { chalcones, } \\
\text { dihydrochalcones, } \\
\text { sugars }\end{array}$ & HSV-1 & $\begin{array}{l}\text { Inhibition of } \\
\text { adsorption in cells at } \\
0.1 \mathrm{mg} / \mathrm{ml} \text { when } \\
\text { incubation was } \geq \\
60 \mathrm{~min}\end{array}$ & $\mathrm{CC}_{50}=0.13 \mathrm{mg} / \mathrm{ml}$ & (87) \\
\hline & & & & $\begin{array}{l}\text { HSV- } \\
1 / \text { HSV-2 }\end{array}$ & $\begin{array}{l}\text { Virucidal effect for } \\
\text { extract } \\
\text { concentration } \geq 10 \\
\mathrm{mg} / \mathrm{ml} \text { and } \\
\text { incubation for at } \\
\text { least } 15 \mathrm{~min}\end{array}$ & & \\
\hline & $\begin{array}{l}\text { Ethanol extract, chloroform soluble } \\
\text { fraction }\end{array}$ & & $\begin{array}{l}\text { Kaempferol, } \\
\text { galangin, quercetin, } \\
\text { fisetin, chrysin, } \\
\text { luteolin, acacetin, } \\
\text { caffeic acid, FA, } \\
\text { p-coumaric acid } \\
\text { (based on literature) }\end{array}$ & HRV-4 & $\begin{array}{l}\mathrm{IC}_{50}=5.0 \mu \mathrm{g} / \mathrm{ml} \\
\text { (SRB method) }\end{array}$ & $\mathrm{CC}_{50}=82.3 \mu \mathrm{g} / \mathrm{ml}$ & (88) \\
\hline & - & & FA (pure compound) & HRV-2 & $\mathrm{IC}_{50}=175.1 \mu \mathrm{M}$ & $\mathrm{CC}_{50}>5,000 \mu \mathrm{M}$ & \\
\hline & & & & HRV-3 & $\mathrm{IC}_{50}=248.7 \mu \mathrm{M}$ & & \\
\hline & & & & HRV-4 & $\mathrm{IC}_{50}=232.3 \mu \mathrm{M}$ & & \\
\hline \multirow[t]{2}{*}{ Strychnos minor } & $\begin{array}{l}\text { Successive extraction of dried plant } \\
\text { with methanol, sonication, vacuum } \\
\text { evaporation }\end{array}$ & Leaves & $\begin{array}{l}\text { Gallic acid, FA, } \\
\text { o-coumaric acid, } \\
\text { quercetin }\end{array}$ & $\mathrm{H} 1 \mathrm{~N} 1$ & $\begin{array}{l}\mathrm{I}_{50}=46.69 \mu \mathrm{g} / \mathrm{ml} \\
\text { (SRB method) }\end{array}$ & $\mathrm{CC}_{50}=1,026 \mu \mathrm{g} / \mathrm{ml}$ & (89) \\
\hline & & Stem & & $\mathrm{H} 1 \mathrm{~N} 1$ & $\mathrm{IC}_{50}=22.43 \mu \mathrm{g} / \mathrm{ml}$ & $\mathrm{CC}_{50}=100 \mu \mathrm{g} / \mathrm{ml}$ & \\
\hline \multirow{2}{*}{$\begin{array}{l}\text { Diotacanthus } \\
\text { albiflorus }\end{array}$} & & Leaves & & $\mathrm{H} 1 \mathrm{~N} 1$ & $I_{50}=60.09 \mu \mathrm{g} / \mathrm{ml}$ & $\mathrm{CC}_{50}=100 \mu \mathrm{g} / \mathrm{ml}$ & \\
\hline & & Stem & & $\mathrm{H} 1 \mathrm{~N} 1$ & $\mathrm{IC}_{50}=33.98 \mu \mathrm{g} / \mathrm{ml}$ & $\mathrm{CC}_{50}=50 \mu \mathrm{g} / \mathrm{ml}$ & \\
\hline
\end{tabular}


TABLE 2 | Continued

\begin{tabular}{|c|c|c|c|c|c|c|c|}
\hline Plant source & Extraction method ${ }^{a}$ & & $\begin{array}{l}\text { Major detected } \\
\text { compounds }\end{array}$ & Virus & Antiviral effect & Cytotoxicity & References \\
\hline \multirow{2}{*}{$\begin{array}{l}\text { Strychnos } \\
\text { nux-vomica }\end{array}$} & & Leaves & & $\mathrm{H} 1 \mathrm{~N} 1$ & $\mathrm{IC}_{50}=33.36 \mu \mathrm{g} / \mathrm{ml}$ & $\mathrm{CC}_{50}=20 \mu \mathrm{g} / \mathrm{ml}$ & \\
\hline & & Stem & & $\mathrm{H} 1 \mathrm{~N} 1$ & $\mathrm{IC}_{50}=23.60 \mu \mathrm{g} / \mathrm{ml}$ & $\mathrm{CC}_{50}=40 \mu \mathrm{g} / \mathrm{ml}$ & \\
\hline \multirow[t]{2}{*}{ Cayratia pedata } & & Leaves & & $\mathrm{H} 1 \mathrm{~N} 1$ & $\mathrm{IC}_{50}=65.99 \mu \mathrm{g} / \mathrm{ml}$ & $\mathrm{CC}_{50}=100 \mu \mathrm{g} / \mathrm{ml}$ & \\
\hline & & Stem & & $\mathrm{H} 1 \mathrm{~N} 1$ & $\mathrm{IC}_{50}=20.50 \mu \mathrm{g} / \mathrm{ml}$ & $\mathrm{CC}_{50}=18.30 \mu \mathrm{g} / \mathrm{ml}$ & \\
\hline \multirow[t]{5}{*}{$\begin{array}{l}\text { Hibiscus sabdariffa } \\
\text { (roselle) }\end{array}$} & \multirow[t]{2}{*}{$\begin{array}{l}\text { Calyses extracted in deionized } \\
\text { distilled boiling water and filtered }\end{array}$} & & \multirow{2}{*}{$\begin{array}{l}\text { Anthocyanins, } \\
\text { phenolic acids, } \\
\text { organic acids, } \\
\text { saponins, and } \\
\text { alkaloids, including } \\
\text { malic acid, } \\
\text { protocatechuic acid } \\
\text { and FA (based on } \\
\text { literature) }\end{array}$} & FCV-F9 & $\begin{array}{l}\text { Viral titer } \\
\text { undetectable after } \\
15 \mathrm{~min} \text { with } 40 \text { or } \\
100 \mathrm{mg} / \mathrm{ml} \\
\text { Viral titer } \\
\text { undetectable after } \\
24 \mathrm{~h} \text { with } 40 \mathrm{mg} / \mathrm{ml}\end{array}$ & \multirow[t]{2}{*}{$\begin{array}{l}\text { Cytotoxicity } \\
\text { observed at > } 40 \\
\mathrm{mg} / \mathrm{ml}\end{array}$} & \multirow[t]{5}{*}{ (90) } \\
\hline & & & & HAV & $\begin{array}{l}\text { Viral titer } \\
\text { undetectable after } \\
24 \mathrm{~h} \text { with } 40 \mathrm{mg} / \mathrm{ml}\end{array}$ & & \\
\hline & \multirow[t]{3}{*}{-} & & FA (pure compound) & FCV-F9 & $\begin{array}{l}\text { Viral titer } \\
\text { undetectable after } \\
15 \mathrm{~min} \text { after } 3 \mathrm{~h} \text { with } \\
0.5 \text { or } 1 \mathrm{mg} / \mathrm{ml}\end{array}$ & \multirow[t]{3}{*}{-} & \\
\hline & & & & MNV-1 & $\begin{array}{l}0.94 \mathrm{log} P F U / \mathrm{ml} \\
\text { reduction after } 24 \mathrm{~h} \\
\text { at } 1 \mathrm{mg} / \mathrm{ml}\end{array}$ & & \\
\hline & & & & HAV & $\begin{array}{l}\text { No significant } \\
\text { reduction in viral } \\
\text { titers after } 24 \mathrm{~h}\end{array}$ & & \\
\hline $\begin{array}{l}\text { Eucaluptus } \\
\text { camaldulensis (river } \\
\text { red gum) }\end{array}$ & $\begin{array}{l}\text { Extraction of dried stem bark with } \\
\text { acetone, filtration and drying by } \\
\text { evaporation }\end{array}$ & & $\begin{array}{l}\text { Benzoic acid, quinol, } \\
\text { myricetin, salicylic } \\
\text { acid, rutin, ellagic } \\
\text { acid, vanillic acid, } \\
\text { p-hydroxybenzoic } \\
\text { acid, chlorogenic } \\
\text { acid, syringic acid, } \\
\text { o-coumaric acid, FA } \\
(4.56 \mathrm{mg} / 100 \mathrm{~g}) \\
\text { gallic acid, vanillin, } \\
\text { cinnamic acid, } \\
\text { p-coumaric acid etc. }\end{array}$ & TMV & $\begin{array}{l}72.22 \% \text { inhibition } \\
\text { (protective effect) } \\
50.43 \% \text { inhibition } \\
\text { (curative effect) } \\
33.33 \% \text { inhibition } \\
\text { (inactivating effect) } \\
\text { at } 100 \mu \mathrm{g} / \mathrm{ml}\end{array}$ & - & (91) \\
\hline
\end{tabular}


TABLE 2 | Continued

\begin{tabular}{|c|c|c|c|c|c|c|}
\hline Plant source & Extraction method ${ }^{a}$ & $\begin{array}{l}\text { Major detected } \\
\text { compounds }\end{array}$ & Virus & Antiviral effect & Cytotoxicity & References \\
\hline $\begin{array}{l}\text { Humulus lupulus } \\
\text { (hops) }\end{array}$ & $\begin{array}{l}\text { Hydroalcoholic extract of female } \\
\text { inflorescences }\end{array}$ & $\begin{array}{l}\text { Chlorogenic acid, } \\
\text { t-FA }(0.078 \mu \mathrm{g} / \mathrm{mg}) \text {, } \\
\text { gallic acid, } p-\mathrm{OH} \\
\text { benzoic acid, rutin, } \\
\text { syringic acid }\end{array}$ & $\begin{array}{l}\text { Influenza } \\
\text { (PR8, } \\
\text { pH1N1, } \\
\text { NWS, } \\
\text { ULSTER) }\end{array}$ & $\begin{array}{l}\mathrm{IC}_{50}=99 \mu \mathrm{g} / \mathrm{ml} \text { for } \\
\text { the PR8 strain }\end{array}$ & $\begin{array}{l}\text { Cell viability > 90\% } \\
\text { for } 20-140 \mu \mathrm{g} / \mathrm{ml}\end{array}$ & (92) \\
\hline $\begin{array}{l}\text { Berberis lyceum } \\
\text { (barberry) }\end{array}$ & $\begin{array}{l}\text { Extraction of leaves with } 70 \% \\
\text { methanol, filtration and drying by } \\
\text { vacuum evaporation }\end{array}$ & $\begin{array}{l}\text { Quercetin, myricetin, } \\
\text { gallic acid, caffeic } \\
\text { acid, FA ( } 0.37 \mathrm{mg} / \mathrm{g} \\
\text { DE) }\end{array}$ & $\mathrm{HCV}$ & $\mathrm{IC}_{50}=11.44 \mu \mathrm{g} / \mathrm{ml}$ & $\mathrm{CC}_{50}>200 \mu \mathrm{g} / \mathrm{ml}$ & (93) \\
\hline \multirow[t]{2}{*}{$\begin{array}{l}\text { Licopersicum } \\
\text { esculentum (tomato) }\end{array}$} & $\begin{array}{l}\text { Leaf extraction in } 95 \% \text { ethanol: } 5 \% \\
\text { acetic acid, filtration and evaporation }\end{array}$ & $\begin{array}{l}\text { Chlorogenic acid, } \\
\text { caffeic acid, FA } \\
(0.0195 \mathrm{mg} / 100 \mathrm{~g} \\
\text { DW), gallic acid, } \\
\text { rutin, quercetin }\end{array}$ & MS2 & $\begin{array}{l}3.47 \text { logPFU/ml } \\
\text { (reduction of viral } \\
\text { titer) }\end{array}$ & - & (94) \\
\hline & & & Av-05 & $5.78 \log \mathrm{PFU} / \mathrm{ml}$ & & \\
\hline \multirow[t]{2}{*}{$\begin{array}{l}\text { Solanum tuberosum } \\
\text { (potato) }\end{array}$} & $\begin{array}{l}\text { Mixing of dried peels with } 95 \% \\
\text { ethanol: } 5 \% \text { acetic acid, filtration, } \\
\text { sonication of the residue with ethanol, } \\
\text { filtration of the sonicated residue, } \\
\text { mixing of the two supernatants and } \\
\text { concentration }\end{array}$ & $\begin{array}{l}\text { Gallic acid, } \\
\text { chlorogenic acid, } \\
\text { caffeic acid, FA ( } 3.29 \\
\text { mg/100 g DW), rutin, } \\
\text { quercetin }\end{array}$ & MS2 & $\begin{array}{l}3.9 \text { logPFU } / \mathrm{ml} \\
\text { (reduction of viral } \\
\text { titer) }\end{array}$ & - & (95) \\
\hline & & & Av-05 & $2.8 \log \mathrm{PFU} / \mathrm{ml}$ & & \\
\hline $\begin{array}{l}\text { Haplophyllum } \\
\text { tuberculatum }\end{array}$ & $\begin{array}{l}\text { Extraction of the whole plant with } \\
\text { ethanol (soaking method), filtration } \\
\text { and evaporation }\end{array}$ & $\begin{array}{l}\text { Gallic acid, FA } \\
\text { ( } 26.86 \mathrm{mg} / \mathrm{kg}) \text {, } \\
\text { catechin, quinol, } \\
\text { syringic acid, caffeic } \\
\text { acid, vanillic acid, } \\
\text { ellagic acid, } \\
\text { cinnamic acid, } \\
\text { o-coumaric acid, } \\
\text { benzoic acid, } \\
\text { p-hydroxy benzoic } \\
\text { acid }\end{array}$ & TMV & $\begin{array}{l}\text { 65.38\% inhibition } \\
\text { (protective effect); } \\
95.73 \% \text { inhibition } \\
\text { (inactivating effect) }\end{array}$ & - & (96) \\
\hline $\begin{array}{l}\text { Garcinia } \\
\text { mangostana } \\
\text { (mangosteen) }\end{array}$ & - & $\begin{array}{l}\text { FA isolated from the } \\
\text { extract }\end{array}$ & $\mathrm{H} 1 \mathrm{~N} 1$ & $\mathrm{IC}_{50}=140 \mu \mathrm{g} / \mathrm{ml}$ & $\mathrm{CC}_{50}=702 \mu \mathrm{M}$ & (78) \\
\hline \multirow[t]{2}{*}{ Rice bran } & - & $\begin{array}{l}\text { Isolated cycloartenol } \\
\text { ferulate }\end{array}$ & HIV-1 & $\begin{array}{l}\mathrm{IC}_{50}=2.2 \mu \mathrm{g} / \mathrm{ml} \\
\text { (reverse } \\
\text { transcriptase } \\
\text { inhibition assay) }\end{array}$ & - & (97) \\
\hline & & $\begin{array}{l}\text { Isolated 24- } \\
\text { methylenecycloartanol } \\
\text { ferulate }\end{array}$ & & & & \\
\hline
\end{tabular}


TABLE 2 | Continued

\begin{tabular}{|c|c|c|c|c|c|c|}
\hline Plant source & Extraction method ${ }^{a}$ & $\begin{array}{l}\text { Major detected } \\
\text { compounds }\end{array}$ & Virus & Antiviral effect & Cytotoxicity & References \\
\hline & & & & $\begin{array}{l}94 \% \text { inhibition (4 } \\
\mathrm{mg} / \mathrm{ml})\end{array}$ & & \\
\hline & - & & $\mathrm{HIV}-1$ & $\mathrm{IC}_{50}=1.9 \mu \mathrm{g} / \mathrm{ml}$ & & \\
\hline & & & & $\begin{array}{l}89 \% \text { inhibition (4 } \\
\mathrm{mg} / \mathrm{ml})\end{array}$ & & \\
\hline \multirow[t]{3}{*}{$\begin{array}{l}\text { Gallesia gorazema } \\
\text { (pau-d'alho) }\end{array}$} & $\begin{array}{l}\text { Soaking of dried plant roots with } \\
\text { dichloromethane }\end{array}$ & $\begin{array}{l}\alpha \text {-Amyrin, } \\
\text { tetradecanal, } \\
\text { palmitic acid, } \\
\text { octadecanal, } \\
\gamma \text {-sitosterol, } \\
\beta \text {-amyrin, 28- } \\
\text { hydroxyoctacosyl } \\
\text { ferulate }\end{array}$ & HSV-1 & $\begin{array}{l}93 \% \text { inhibition } \\
\text { (protective effect at } \\
100 \mu \mathrm{g} / \mathrm{ml})\end{array}$ & $\mathrm{CC}_{50}>200 \mu \mathrm{g} / \mathrm{ml}$ & (98) \\
\hline & - & $\begin{array}{l}28- \\
\text { hydroxyoctacosyl } \\
\text { ferulate isolated from } \\
\text { extract }\end{array}$ & HSV-1 & $\mathrm{EC}_{50}=21.6 \mu \mathrm{g} / \mathrm{ml}$ & $\mathrm{CC}_{50}>200 \mu \mathrm{g} / \mathrm{ml}$ & \\
\hline & & & HSV-2 & $\mathrm{EC}_{50}=86.5 \mu \mathrm{g} / \mathrm{ml}$ & & \\
\hline $\begin{array}{l}\text { Vaccinium oldhamii } \\
\text { (wild blueberry) }\end{array}$ & $\begin{array}{l}\text { Extraction of dried fruits with } 80 \% \\
\text { ethanol, concentration and elution } \\
\text { with } 40 \% \text { ethanol through column } \\
\text { chromatography }\end{array}$ & $\begin{array}{l}\text { Procyanidin B2, } \\
\text { unspecified ferulic } \\
\text { acid derivatives, two } \\
\text { ferulic acid } \\
\text { O-hexosides, } \\
\text { quercetin-3-O- } \\
\text { rhamnoside and } \\
\text { quercetin-O- } \\
\text { pentoside-O- } \\
\text { rhamnoside }\end{array}$ & IFV & $\begin{array}{l}\mathrm{IC}_{50}=22 \mu \mathrm{g} / \mathrm{ml} \\
\text { (viral inhibition } \\
\text { plaque assay) }\end{array}$ & $\mathrm{CC}_{50}=160 \mu \mathrm{g} / \mathrm{ml}$ & (99) \\
\hline
\end{tabular}

${ }^{a}$ Conditions or fractions that offer highest antiviral effect in the respective study; HSV, herpes simplex virus; RSV, respiratory syncytial virus; ADV, adenovirus; ECV, echovirus; EV, enterovirus; CV, coxsackievirus; PPRSV, porcine reproductive and respiratory syndrome virus; HRV, human rhinovirus; H1N1, influenza A virus subtype; FCV, feline calicivirus; MNV, murine noronivirus; HAV, hepatitis A virus; TMV, tobacco mosaic virus; HCV, hepatitis $C$ virus; MS2, Av-05, bacteriophage; HIV, human immunodeficiency virus; IFV, influenza virus; $E C_{50}$, half-maximal effective concentration; the concentration required to obtain a $50 \%$ inhibitory effect, IC $C_{50}$, half-maximal inhibitory concentration; the concentration at which the number of viral plaques is reduced by $50 \%, C C_{50}$, half-maximal cytotoxicity concentration, the concentration that reduced cell viability to $50 \%$ of the control; DW, dry weight; DE, dry extract. 
TABLE 3 | Antiviral effect of FA derivatives.

Derivative
Virus

HIV-1<smiles>COc1cc(/C=C(\C(=O)O)c2cc(/C=C/C(=O)O)cc(OC)c2O)ccc1O</smiles>

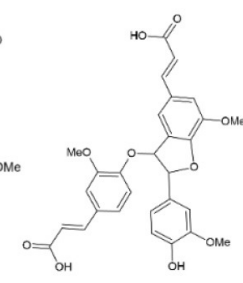

FA amide of 3-aminomethyl glaucine

FA amides<smiles></smiles><smiles>COc1cccc(/C=C/C(=O)NCC(O[Tl])c2cccc(O)c2)c1</smiles>

Hydrogenated FA amides<smiles>[R]OC(CNC(=O)CCc1ccc(O)c(OC)c1)c1cccc(CCNC(=O)CCc2ccc(O)c(OC)c2)c1</smiles>

$\alpha, \beta$-unsaturated amide derivatives of FA with an $\alpha$ aminophosphonate moiety

TMV

TMV
Example: diferulate, triferulate
Antiviral activity/Cytotoxicity of the compound

$$
\begin{aligned}
& I C_{50}=1.0 \mu \mathrm{g} / \mathrm{ml} \\
& I_{50}=0.9 \mu \mathrm{g} / \mathrm{ml} \\
& I_{50}=2.7 \mu \mathrm{g} / \mathrm{ml} \\
& I_{50}=3.2 \mu \mathrm{g} / \mathrm{ml}
\end{aligned}
$$

$>30 \mathrm{kDa}$

30-10 kDa

$10-1 \mathrm{kDa}$

$1 \mathrm{kDa}-500 \mathrm{Da}$
Structure

References

HRV-14

$$
\mathrm{IC}_{50}=12.00 \mu \mathrm{M}
$$$$
\mathrm{CC}_{50}=113 \mu \mathrm{M}
$$

CMV

TMV<smiles>[R]C=NNC(=O)/C=C/c1ccc2c(c1)OCCP2</smiles>

acylhydrazon

moiety

FA sulfonamides<smiles>[R]OC(=O)/C=C/c1ccc(OCCNS([R])(=O)=O)c(OC)c1</smiles>

TMV

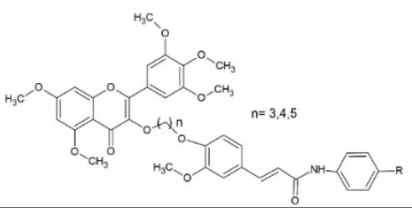

$40.7 \%$ protective effect $46.4 \%$ curative effect

$27.3 \%$ protective effect $30.4 \%$ curative effect

$15.2 \%$ protective effect $28.9 \%$ curative effect

$\mathrm{R}^{1}: \mathrm{n}-\mathrm{Pr} ;$

$\mathrm{R}^{2}: \mathrm{H}$

$\mathrm{R}^{3}$ : propynyl

$\mathrm{R}^{1}: \mathrm{Me}$

$\mathrm{R}^{2}: p-\mathrm{Cl}$

$\mathrm{R}^{3}$ : propynyl

$\mathrm{R}^{1}: p$-benzyloxy

$\mathrm{R}^{2}$ : propynyl

$\mathrm{R}^{1}: 4-\mathrm{Cl}$

$\mathrm{EC}_{50}$
effect)

$\mathrm{EC}_{50}=285.42 \mu \mathrm{g} / \mathrm{ml}$ (curative effect)

$\mathrm{EC}_{50}=216.30 \mu \mathrm{g} / \mathrm{ml}$ (protective effect)

$\mathrm{EC}_{50}=284.67 \mu \mathrm{g} / \mathrm{ml}$ (curative effect)

$18.8 \%$ curative effect

$\mathrm{R}^{1}: \mathrm{PhCH}_{2}$

$23.0 \%$ protective effect

$94.2 \%$ inactivating effect

$\mathrm{EC}_{50}=36.59 \mu \mathrm{g} / \mathrm{ml}$

$39.8 \%$ curative effect

$59.7 \%$ protective effect

$87.3 \%$ inactivating effect

$\mathrm{R}^{1}:-\mathrm{C}_{2} \mathrm{H}_{5}$

$\mathrm{R}^{2}: 4-\mathrm{NO}_{2}-\mathrm{Ph}$

$\mathrm{EC}_{50}=84.80 \mu \mathrm{g} / \mathrm{ml}$

$55.5 \%$ curative effect

$53.3 \%$ protective effect

$\mathrm{R}: 4-\mathrm{Br}, n=3$ with an FA amide scaffold

$\mathrm{EC}_{50}=472.4 \mu \mathrm{g} / \mathrm{ml}$ 
TABLE 3 | Continued

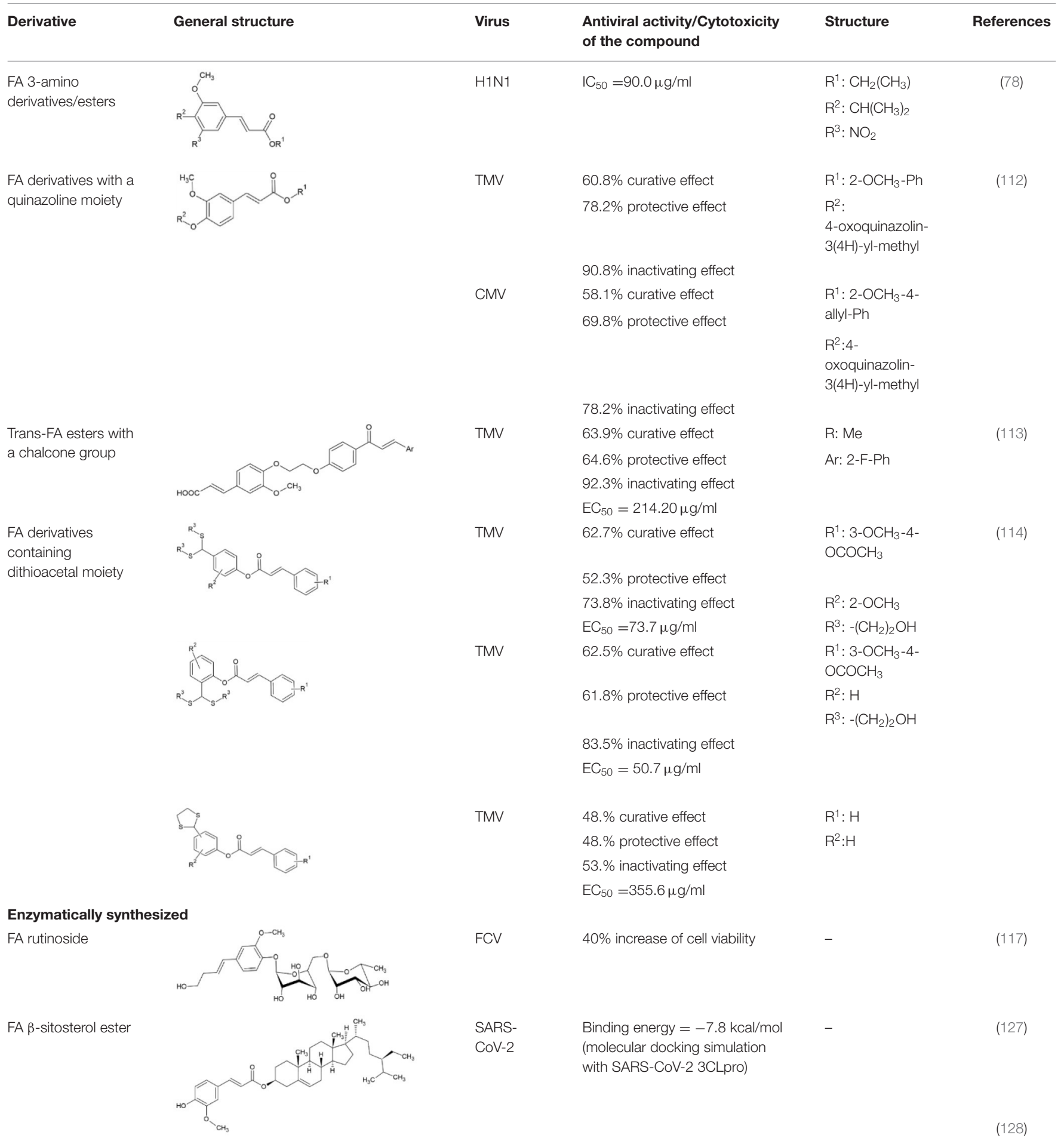

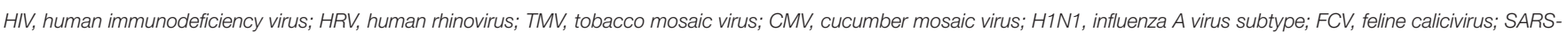

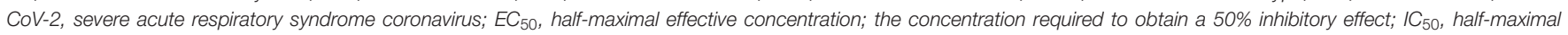

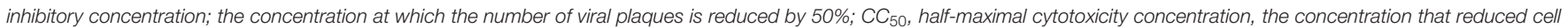
viability to $50 \%$ of the control. 
Ranunculaceae), including C. heracleifolia and C. dahurica has been used traditionally as an antipyretic, analgesic, antiinflammatory agent in East Asian countries (103).

The herbaceous perennial broadleaf plantain (Plantago major L.; Plantaginaceae) is popular in traditional Chinese medicine and has long been used for treating various diseases varying from cold to viral hepatitis. The aqueous extract of the whole plant, along with pure compounds representing the five main classes of biologically active compounds found in its extracts (benzoic acids, flavonoids, iridoid glycoside, hydroxycinnamic acids and triterpenes), have been investigated for their ability to suppress herpesviruses and adenoviruses (ADV). Interestingly, the aqueous extract of the whole plant showed only inhibitory activity against HSV-2 while pure FA was promising against ADV-8 and ADV-11, showing an $\mathrm{EC}_{50}=52.5$ and $23.3 \mu \mathrm{g} / \mathrm{ml}$ respectively, evaluated with the XTT assay on basal cell carcinoma cells. Among hydroxycinnamic acids, caffeic acid was the most active, achieving inhibition for HSV-1, HSV2 and ADV-3 but not ADV-8 and ADV-11, followed by chlorogenic acid, which showed activity against all the mentioned viruses (83).

FA was also highlighted to be the major constituent in hexanic and hexane-ethyl acetate extracts of fig latex (Ficus carica; Moraceae). The two extracts proved to inhibit viral entry into Vero cells, since they were incubated with them prior to inoculation with HSV-1, echovirus type 11 (ECV-11) and ADV. Inhibition of the virus was successful also when the extracts were incubated with infected cells, as well as when they were incubated with the virus and then brought in contact with the cells. Thus, F. carica extracts could block viral DNA (for HSV-1 and ADV) and RNA (for ECV-11) replication and also exhibit virucidal activity (84).

Kalanchoe (Crassulaceae) are succulent plants mainly native to Madagascar and tropical Africa. In traditional medicine, Kalanchoe species have been used to treat infections, rheumatism and inflammation. Leaf extract from Kalanchoe gracilis, containing $7.59 \% \mathrm{w} / \mathrm{w}$ FA, exhibited antiviral activity against RNA enteroviruses enterovirus 71 (EV71) and coxsackievirus A16 (CVA16) that was demonstrated by an half maximal inhibitory concentration $\left(\mathrm{IC}_{50}\right)$ of 35.88 and $42.91 \mu \mathrm{g} / \mathrm{ml}$, respectively, using the plaque reduction assay on $\mathrm{RD}$ cells. Further in vitro investigation revealed that the leaf extract achieved inhibition of viral protease $2 \mathrm{~A}$ that is present in both viruses, showing an $\mathrm{IC}_{50}$ value of $40.82 \mu \mathrm{g} / \mathrm{ml}$ for EV71 and $47.87 \mu \mathrm{g} / \mathrm{ml}$ for CVA16 employing the cell-based FRET assay (85). Another plant with antiviral properties in whose extract FA has been detected as a secondary metabolite, is Eugenia singampattiana, a critically endangered small tree belonging to the Myrtaceae family that is endemic in India. Extracts of the leaves were used to treat Marc-145 cells infected with porcine reproductive and respiratory syndrome virus (PRRSV). The methanol and water extracts achieved suppression of the virus at a concentration of 25 and 75-100 $\mu$ g, respectively (86).

Two bacteriophages used as surrogates for human enteric viruses, MS2 and Av-05, were inhibited by tomato extracts (leaf, stems, roots, whole plant) containing FA, although gallic acid was the major identified phenolic acid. Virucidal assay using $5 \mathrm{mg} / \mathrm{ml}$ of the leaf extracts resulted in 3.47 and $5.78 \log \mathrm{PFU} / \mathrm{ml}$ reduction of MS2 and Av-05 titers respectively for Pitenza cultivar, and 3.78 and $4.93 \log \mathrm{PFU} / \mathrm{ml}$ reduction of the viral titers for Floradade cultivar (94). Potato peel (Solanum tuberosum) acidified ethanol extract, containing $3.29 \mathrm{mg}$ FA/100 g dry weight, was also tested with a standardized plaque assay against the two bacteriophages and reduced viral titer by $3.9 \log \mathrm{PFU} / \mathrm{ml}$ for MS2 and 2.8 $\log \mathrm{PFU} / \mathrm{ml}$ for Av-05 when incubated with the viruses for $3 \mathrm{~h}$ with $5 \mathrm{mg} / \mathrm{ml}$ (95).

The aqueous extract of the red calyces of Hibiscus sabdariffa (roselle; Malvaceae) has achieved undetectable viral titers at a concentration of $40 \mathrm{mg} / \mathrm{ml}$, when tested against feline calicivirus (FCV-F9), murine norovirus (MNV-1) and hepatitis A virus (HAV). FA was also tested separately, together with protocatechuic acid and malic acid as main components of the extract. FA at a concentration of $1 \mathrm{mg} / \mathrm{ml}$ exhibited promising results, inhibiting two viruses to the point where they could not be detected after $3 \mathrm{~h}$ (FCV-F9) and $24 \mathrm{~h}$ (MNV-1). However, FA did not have an inhibitory effect against HAV (90). Methanolic leaf extract of the medicinal plant Berberis lyceum (barberry; Berberidaceae) containing FA, successfully inhibited replication of hepatitis $\mathrm{C}$ virus $(\mathrm{HCV})$, with $\mathrm{IC}_{50}=11.44 \mu \mathrm{g} / \mathrm{ml}$ calculated in infected HepG2 cells. The extract also seems to significantly inhibit the expression of the viral non-structural protein NS5A by 42 and $61 \%$ at 100 and $200 \mu \mathrm{g} / \mathrm{ml}$ (93).

Eucaluptus camaldulensis bark extract, containing 4.56 $\mathrm{mg} / 100 \mathrm{~g}$ FA among other phenolic acids and flavonoids, was able to protect, cure from and inactivate tobacco mosaic virus (TMV), as determined utilizing the half leaf method on Nicotiana glutinosa leaves. When used to treat the leaves at a concentration of $100 \mu \mathrm{g} / \mathrm{ml} 24 \mathrm{~h}$ before infection, the extract offered $72.2 \%$ inhibition of the virus. Although the most promising results yielded for the protective effect of the extract, it also had curative and inactivating antiviral action, with the respective inhibition percentages reaching 50.43 and $33.33 \%$ (91). In another study by the same research group, ethanol extract of the whole plant from Haplophyllum tuberculatum (Rutaceae) had both protective and inactivating effect for TMV (96). The extract contained FA among other phenolic acids.

FA is also one of the major phenolic compounds in the hydroalcoholic extract of Humulus lupulus (hops) that was found active against various strains of the influenza virus (PR8, pH1N1, NWS, ULSTER). Antiviral assays performed on MadinDarby Canine Kidney (MDCK) and A549 lung carcinoma cells showed considerable potential of the plant extract, as it inhibits replication of the PR8 strain with an $\mathrm{IC}_{50}$ of $99 \mu \mathrm{g} / \mathrm{ml}$. Anti-influenza effect was tested when the extract was added to the culture in different stages of infection (before, during and after). Viral replication was partly inhibited when virus was incubated with extract before infection, suggesting a direct effect on the virus. By adding extract to the cells during the infection, a significant reduction of the viral titer was noticed for PR8, NWS and ULSTER strains (46, 50 and 29\% respectively). Addition after infection resulted in 75, 44 and $29 \%$ inhibition, respectively. The highest inhibitory potential, against all the viral strains examined, was calculated in the cases where the extract was present in all the phases of the 
infection (92). It was speculated that since the extract was able to restore the reducing conditions of infected cells, by increasing glutathione content, its antiviral activity might be also due to an interference with redox-sensitive pathways required for viral replication.

In vitro studies in MDCK cells, testing the antiviral activity of methanol extracts of medicinal plants from South India against influenza A virus subtype $\mathrm{H} 1 \mathrm{~N} 1$, indicate promising properties of leaf and stem extracts from Strychnos minor, Diotacanthus albiflorus, Strychnos nux-vomica and Cayratia pedata. The extracts mainly comprised of gallic acid, FA, $o$-coumaric acid and quercetin. The metabolite content was predominantly different among different samples, while although total phenolic content was related to positive influence on viral inhibition, a negative correlation of FA concentration was considered in respect with antiviral activity (89). Hariono et al. (78) also report inhibitory activity of FA isolated from Garcinia mangostana (mangosteen; Clusiaceae) against the neuraminidase of $\mathrm{H} 1 \mathrm{~N} 1$ $\left(\mathrm{IC}_{50}=140 \mu \mathrm{M}\right)$. Mangosteen has been known in traditional medicine mostly in Southeast Asia.

Although not a plant extract, propolis, that incorporates exudates gathered from tree buds, sap flows or other botanical sources, has been shown to contain FA as an important constituent. A patented ethanol extract from Canadian propolis, that was harvested from beehives located in an area rich in poplar trees, has been shown to be effective toward HSV-1 and HSV-2 when tested for its prevention of viral adsorption in MDBK cells and virucidal effect (87). The anti-rhinovirus activity of Brazilian propolis and its constituents has also been evaluated. Among various fractions of propolis ethanol extract, the replication of human rhinovirus type 4 (HRV-4) in HeLa cells was limited using a chloroform- and ethyl acetate-soluble fraction by $\mathrm{IC}_{50}$ $=5.0$ and $5.2 \mu \mathrm{g} / \mathrm{ml}$ respectively (88). In the same study, pure FA inhibited three HRV types with an $\mathrm{IC}_{50}$ ranging between 175.1 and $248.7 \mu \mathrm{M}$, showing better performance than the tested drug reference ribarivin. Phenolic acids, such as caffeic and $p$ coumaric acid, as well as flavonols and flavonones had also superior inhibitory effect.

FA derivatives with antiviral activity has been isolated from plant sources. For example, cycloartenol ferulate and 24methylenecycloartanol ferulate are two compounds found in rice bran that effectively inhibit human immunodeficiency virus 1 $(\mathrm{HIV}-1)$ reverse transcriptase, with a considerable $\mathrm{IC}_{50}$ value of $2.2 \mu \mathrm{M}$ and $1.9 \mu \mathrm{M}$, respectively (97). Moreover, De Jesus Silva Júnior et al. (98) have isolated an FA derivative identified as 28-hydroxyoctacosyl ferulate from the dichloromethane root extract of Gallesia gorazema (Petiveriaceae), a shrub widely distributed in the Atlantic Rain Forest of Brazil. The ester was tested against HSV-1 and HSV-2 viruses, inhibiting both by 99.8 and $97.8 \%$ and with a half-maximal cytotoxicity concentration equal to 21.6 and $86.5 \mu \mathrm{g} / \mathrm{ml}$, respectively, whereas the crude root extract inhibited only HSV-1 by $93 \%$. The ester shows a much higher selectivity for HSV-1 than HSV-2, which could be a strong indication that it is responsible for the antiviral activity of the extract against HSV-1. Unspecified FA derivatives are pointed out as major contributors to the anti-influenza activity of an extract from Japanese wild blueberries (Vaccinium oldhamii; Ericaceae), preventing the adsorption or cell entry of the virus in MDCK cell cultures. Dry fruit extracts purified through column chromatography with different percentages of ethanol were screened for their antiviral activity and the lowest adsorption inhibitory concentration was calculated to have an $\mathrm{IC}_{50}$ value of $22 \mu \mathrm{g} / \mathrm{ml}$ (99).

\section{FA DERIVATIVES AS ANTIVIRAL AGENTS}

\section{Chemically Synthesized Derivatives}

FA derivatives could enhance the bioactive property of the molecule and potentially influence the distribution and time of stay in the human body. The first reported attempt on chemical derivatization of FA targeting enhancement of its antiviral activity was based on a polymerization strategy to create dehydrogenated polymers that resemble natural structures like lignin. Different size synthesized polymers of FA and $p$ coumaric acid showed antiviral activity against HIV-1 through two mechanisms; by inhibiting cytopathicity induced by the virus and blocking its protease, as examined in MT- 4 cells. The four FA polymer fractions, with a size range of $500 \mathrm{Da}$ to over $30 \mathrm{kDa}$, exhibited HIV-1 protease inhibition with $\mathrm{IC}_{50}$ values between 3.2 and $0.9-1.0 \mu \mathrm{g} / \mathrm{ml}$. It was noticed that the larger the polymer, the lower the $\mathrm{IC}_{50}$ value of the compound (104).

Later, several strategies on FA amidation were explored. Spasova et al. (105) recruited peptide chemistry using coupling reagent $\mathrm{EDC} / \mathrm{HOBt}$ to create amides of 3-aminomethyl glaucine linked to HCAs, including FA. The derivatives were screened for their activity against enteroviruses Poliovirus type 1 (LSc-2ab), CV-B1 and EV-13 and HRV-14. Overall, all 3aminomethylglaucine derivatives of FA, sinapic, $o$ - and $p$ coumaric acids proved to have antiviral properties against HRV14 and were not cytotoxic at respective $\mathrm{IC}_{50}$ concentrations, worthing further investigation.

Huang et al. (106) synthesized a series of substituted FA amide and the corresponding hydrogenated derivatives targeting increased antiviral effect against plant viruses and in particular TMV. Among compounds from both series, the ones that preserved the double bond in the carbon chain had higher activity overall. The best generated compound had protective and curative activity of 40.7 and $46.4 \%$ respectively, in infected N. tabacum leaves. Cui et al. (107) also tested very similar hydrogenated FA derivatives that were synthesized chemically via hydrogenation of $\mathrm{FA}$ using $\mathrm{Pd} / \mathrm{C}$ as catalyst, to produce hydrogenated ethyl ferulate as intermediate, and subsequent substitution with 2-amino-1-phenylethanol derivatives. The two most active compounds had $p$-benzyloxy substitution in the $\mathrm{R}^{1}$ position and propynyl or allyl substitution in the $\mathrm{R}^{2}$ position respectively. They resulted in a protective effect against TMV of 15.2 and $22.8 \%$, respectively and a respective curative effect of 28.9 and $20.7 \%$.

Activity against the two plant viruses, TMV and cucumber mosaic virus (CMV) has been detected in $\alpha, \beta$-unsaturated amide derivatives of FA with an $\alpha$-aminophosphonate moiety. $\mathrm{EC}_{50}$ was observed to be 216.30 and $284.6 \mu \mathrm{g} / \mathrm{ml}$ for TMV and CMV respectively, for a compound with $\mathrm{R}^{1}=4-\mathrm{Cl}$ and $\mathrm{R}^{2}$ $=4-\mathrm{CF}_{3}-\mathrm{Ph}$, which was superior to that of ningnanmycin. All 26 synthesized compounds showed antiviral activity to some level, which was observed to be reinforced by the presence 
of an electron- withdrawing group at the 2- or 4- position of the aromatic ring (108). TMV has also been the target for synthesis of 23 conjugates containing an acylhydrazone moiety that were tested in vivo against the virus and a concentration of $500 \mu \mathrm{g} / \mathrm{ml}$. The majority of them exhibited better inactivating activity than the commercial drug ribavirin. Protective activity ranged from 14.5 to $58.7 \%$, curative activity from 17.4 to $54.2 \%$ and inactivating activity from 59.3 to $94.2 \%$. The compound with the most prominent properties was the one with $\mathrm{R}^{1}=$ $\mathrm{PhCH}_{2}$ and $\mathrm{R}^{2}=2$-Th substitutions, as it had $18.8 \%$ protective, $23.0 \%$ curative, $94.2 \%$ inactivating activity and $\mathrm{EC}_{50}$ equal to $36.59 \mu \mathrm{g} / \mathrm{ml}$ regarding its inactivating activity; almost three times lower than ribavirin (109).

FA sulfonamides are very potent against tobacco mosaic virus (TMV). Antiviral assay conducted with the half-leaf method resulted in curative activity ranging between 30.2-57.9 \%, protection activity between $31.2-59.7 \%$ and inactivation activity $43.7-87.3 \%$ for the different FA derivatives at a concentration of $500 \mu \mathrm{g} / \mathrm{ml}$. The most prominent compound inactivated TMV with an $\mathrm{EC}_{50}$ of $84.8 \mu \mathrm{g} / \mathrm{ml}$, significantly lower than the one of widely used drug ribavirin. Results showed that, as far as structure is concerned, electropositive substituents of the 4-position of the sulfonamide benzene ring are more active than electronegative when it comes to curating effect, whereas the opposite is observed in terms of protection from the virus (110).

Tang et al. (111) have chemically synthesized myricetin derivatives with an FA amide substitution and investigated their activity against TMV. The curative and protective effect of the conjugates at $500 \mu \mathrm{g} / \mathrm{ml}$ was measured using the half-leaf method on $N$. tabacum L. leaves. Results varied, from 15.8 to $55.5 \%$ for the curative effect and 5.3 to $62.1 \%$ for the protective effect of the different derivatives. Further testing for the most promising compounds using preliminary bioassays resulted in calculations of $\mathrm{EC}_{50}$ values of $196.11,425.3$ and $386.7 \mu \mathrm{g} / \mathrm{ml}$ for the protection activity of the best compounds, with the $\mathrm{R}$ substitutions being 3,4-di- $\mathrm{OCH}_{3}, 3,4$-di- $\mathrm{Cl}$ and 4- $\mathrm{Br}$, respectively $(n=3)$. The compound with the $4-\mathrm{Br}$ substitution also had an $\mathrm{EC}_{50}=472.4 \mu \mathrm{g} / \mathrm{ml}$ regarding its curative activity. All the aforementioned results were comparable to commercial antiviral agent ningnanmycin. FA amino derivatives have been tested against their inhibitory activity of $\mathrm{H} 1 \mathrm{~N} 1$ neuraminidase, showing encouraging results. More specifically, pure FA has an inhibitory effect described by $\mathrm{IC}_{50}=140 \mu \mathrm{M}$, while when nitrate substitutes $\mathrm{C} 3, \mathrm{IC}_{50}$ is reduced to $127 \mu \mathrm{M}$. The best results $\left(\mathrm{IC}_{50}=90 \mu \mathrm{M}\right)$ were provided by an ethyl FA ester, with an isopropyl substitution at $\mathrm{C} 2$ and a nitrate at $\mathrm{C} 3(78)$.

FA esters with a quinazoline group are also effective against TMV and CMV when tested in vivo on N. tabacum leaves and Chenopodium amaranticolor leaves, respectively. Overall, the most effective compound was the one with the following substitutions: $\mathrm{R}^{1}=2$ - $\mathrm{OCH}_{3}-\mathrm{Ph}$ and $\mathrm{R}^{2}=4$-oxoquinazolin$3(4 \mathrm{H})$-yl-methyl. It showed $60.8,78.2$ and $90.8 \%$ curative, protective and inactivating activity against TMV and 58.1, 69.8 and $78.2 \%$ for CMV respectively (112). Synthesis and screening of 35 designed trans-FA esters with a chalcone group for their anti-TMV activity was performed by Gan et al. (113). The results were encouraging, as 5 compounds showed better curative and inactivating effect and 7 compounds had higher protective activity than commercially used antivirals, when tested at a concentration of $500 \mu \mathrm{g} / \mathrm{ml}$ with an antiviral biological assay on $N$. tabacum. FA is also reported as a precursor for the chemical synthesis of derivatives containing a dithioacetal moiety. A series of such compounds has been tested for their activity against TMV, and the most successful hit achieved higher antiviral activity than commercial virucidal ribavirin $(62.5 \%$ curative, $61.8 \%$ protective and $83.5 \%$ inactivating activity at a concentration of $500 \mu \mathrm{g} / \mathrm{ml})(114)$.

\section{Potential for Enzymatic Derivatization}

So far, the vast majority of FA conjugation methods has been based on chemical synthesis routes. However, a major drawback of these methods is the requirement for toxic or expensive catalysts, strong acids, high temperatures and long reaction times, resulting in low yields that may not be possible to compensate for the related energy cost (115). In addition, the heat sensitivity and oxidation susceptibility of FA makes such processes more difficult to control (116). Enzymatic derivatization is a developing field of research that introduces a more sustainable solution, applying milder conditions and resulting in exceptional selectivity, due to the use of enzymes as biocatalysts.

A demonstrated route for enzymatic FA derivatization, targeting enhancement of antiviral activity, is via (trans)glycosylation. The only current example is the use of rutinase, an enzyme that naturally catalyzes the hydrolyzation of rutin to quercetin and rutinose, but it can be used for the reverse reaction, aiming to the formation of glycoside derivatives. Katayama et al. (117) have studied the enzymatic synthesis of phenolic acid rutinosides aided by a rutinase derived from tartary buckwheat seeds. Although among vanillic, sinapic, FA and caffeic acid rutinosides, sinapic acid rutinoside had the highest antiviral activity. FA rutinoside also exhibited antiviral activity against FCV in an antiviral assay conducted with the MTT method, preventing the reduction of cell viability after infection. In all cases, conjugation of the phenolic acids with rutin enhanced their anti-FCV activity. This reaction has demonstrated that, since use of FA is often limited by weak solubility in either hydrophilic or hydrophobic formulations, glycosylation with a more hydrophilic compound can be a potent strategy to alter its solubility and cell penetration ability, as well as its biological activity.

A potential route for the enzymatic derivatization of FA resulting in enhancement of antiviral properties is via (trans)esterification. Triaglycerol lipases (EC 3.1.1.3) are most commonly used due to their broad specificity toward lipids and extraordinary robustness in low water media, the latter being required for the formation of esters over hydrolysis (118). There are various reports on the synthesis of FA-based esters employing lipases, including the acylation of FA with ethanol (119), trinolein or trilinolenin (120), glycerol $(121,122)$, arbutin (123), vitamin E (124), phytosterols (125), quercetin (126) and $\beta$-sitosterol (127). In particular, $\beta$-sitosteryl ferulate is mentioned as a potential inhibitor of SARS-CoV-2 main protease 3CLpro, where molecular docking simulations showed a promising binding mode and a binding energy of $-7.8 \mathrm{kcal} / \mathrm{mol}$ for the esterprotease complexes (128). Feruloyl esterases (EC 3.1.1.73) consist another interesting esterase subclass that, in nature, catalyze the 


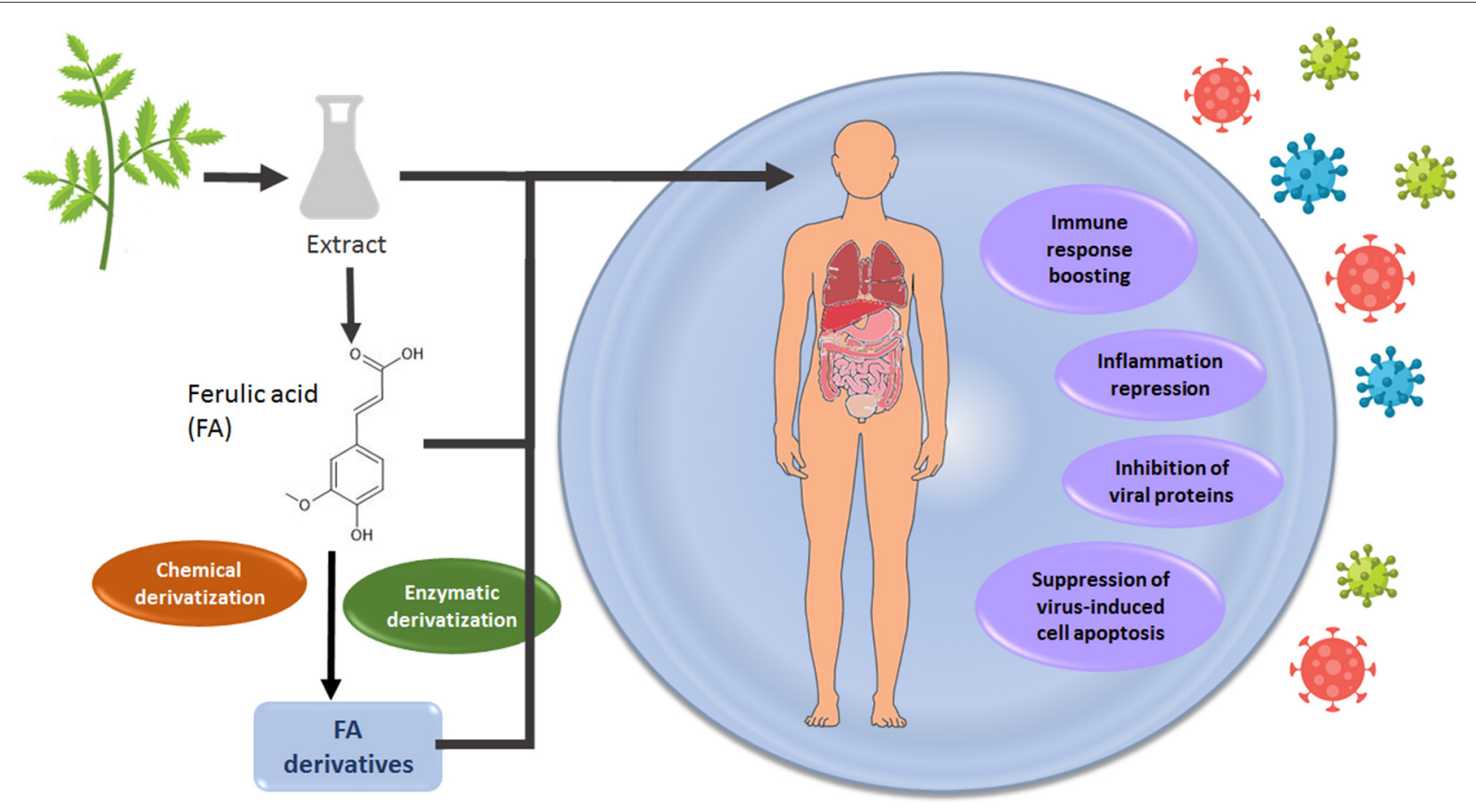

FIGURE 3 | A route for FA valorization toward developing antiviral agents.

hydrolysis of the ester bond between $\alpha$-Larabinofuranose and FA or other hydroxycinnamic acids, present in lignocellulose $(129,130)$. Although they are not so stable in low-water content media as lipases (131), their ability as biocatalytic tools for (trans)esterification has been demonstrated. Their major advantage over lipases is the specificity toward hydroxycinnamic acids, allowing acylation of FA with a wide range of hydrophilic or lipophilic substitutions, such as alcohols, polyols or sugars (132-137).

Last, a promising route is via oxidation/crosslinking employing laccases (EC 1.10.3.2), which oxidize phenols to produce phenoxy radicals that are then polymerized. The oligomers or polymers that occur as products have been reported to have enhanced antioxidant properties (138) and can be a promising alternative to chemical polymerization (104).

Although products from the aforementioned proposed routes have not been evaluated yet in vitro for their antiviral potential, such synthesis routes may result in a highly diverse portfolio of FA based antiviral agents, offering beneficial effects toward boosting our immunity against viral infections. Overall, there are a lot of possibilities for enzymatic modification of FA that not only may enhance its antiviral potential, but also potentially enable a different fate in the metabolic route, having an impact

\section{REFERENCES}

1. Bharadwaj S, Dubey A, Yadava U, Mishra SK, Kang SG, Dwivedi VD. Exploration of natural compounds with anti-SARS-CoV-2 activity via on its absorption site and retention time in the human body. A summary of the synthesized FA derivatives assessed for their antiviral potential is presented in Table 3 .

\section{CONCLUSIONS}

Extraction of FA from plant biomass and its residues and further derivatization for nutraceutical applications could be a viable and high-added value pathway within biorefineries. More systematic studies are expected as a continuation of in vitro studies, in order to confirm the therapeutic effect and distribution of FA derivatives to protect target cells against viruses. Nevertheless, the use of nutraceuticals, such as FA, may aid toward boosting immunity and aid protection from viral infections, which can be a valuable asset for future epidemics and new viruses. An overview of the potential route for FA valorization is presented in Figure 3.

\section{AUTHOR CONTRIBUTIONS}

IA and ES conceptualized and wrote the review paper. UR and PC conceptualized the review paper and provided feedback on the manuscript. All authors contributed to the article and approved the submitted version. inhibition of SARS-CoV-2 Mpro. Brief Bioinform. (2021) 22:1361-77. doi: 10.1093/bib/bbaa382

2. Gordon DE, Jang GM, Bouhaddou $\mathrm{M}$, Xu J, Obernier $\mathrm{K}$, White $\mathrm{KM}$, et al. A SARS-CoV-2 protein interaction map reveals targets for 
drug repurposing. Nature. (2020) 583:459-68. doi: 10.1038/s41586-0202286-9

3. Srinivasan M, Sudheer AR, Menon VP. Ferulic acid: therapeutic potential through its antioxidant property. J Clin Biochem Nutr. (2007) 40:92-100. doi: $10.3164 /$ jcbn. 40.92

4. Antonopoulou I, Varriale S, Topakas E, Rova U, Christakopoulos P, Faraco V. Enzymatic synthesis of bioactive compounds with high potential for cosmeceutical application. Appl Microbiol Biotechnol. (2016) 100:6519-43. doi: 10.1007/s00253-016-7647-9

5. Appiah-Opong R, Commandeur JNM, van Vugt-Lussenburg B, Vermeulen NPE. Inhibition of human recombinant cytochrome P450s by curcumin and curcumin decomposition products. Toxicology. (2007) 235(1-2), 83-91. doi: 10.1016/j.tox.2007.03.007

6. Kumar N, Pruthi V. Potential applications of ferulic acid from natural sources. Biotechnol Rep. (2014) 4:86-93. doi: 10.1016/j.btre.2014.09.002

7. Mueller-Harvey I, Hartley RD, Harris PJ, Curzon EH. Linkage of pcoumaroyl and feruloyl groups to cell-wall polysaccharides of barley straw. Carbohydr Res. (1986) 148:71-85. doi: 10.1016/0008-6215(86)80038-6

8. Colquhoun IJ, Ralet MC, Thibault JF, Faulds CB, Williamson G. Structure identification of feruloylated oligosaccharides from sugarbeet pulp by NMR spectroscopy. Carbohydr Res. (1994) 263:243-56. doi: 10.1016/0008-6215(94)00176-6

9. Ishii $T$, Hiroi $T$. Linkage of phenolic acids to cell-wall polysaccharides of bamboo shoot. Carbohydr Res. (1990) 206:297-310. doi: 10.1016/0008-6215(90)80069-F

10. Waldron KW, Parr AJ, Ng A, Ralph J. Cell wall esterified phenolic dimers: Identification and quantification by reverse phase high performance liquid chromatography and diode array detection. Phytochem Anal. (1996) 7:30512. doi: 10.1002/(SICI)1099-1565(199611)7:6<305::AID-PCA320>3.0.CO;2A

11. Bunzel M, Ralph J, Brüning P, Steinhart H. Structural identification of dehydrotriferulic and dehydrotetraferulic acids isolated from insoluble maize bran fiber. J Agric Food Chem. (2006) 54:6409-18. doi: 10.1021/jf0 $61196 a$

12. Carnachan SM, Harris PJ. Ferulic acid is bound to the primary cell walls of all gymnosperm families. Biochem Syst Ecol. (2000) 28:865-79. doi: 10.1016/S0305-1978(00)00009-0

13. Truong HT, Do Van M, Huynh LD, Nguyen LT, Do Tuan A, Thanh TLX, et al. A method for ferulic acid production from rice bran oil soapstock using a homogenous system. Appl Sci. (2017) 7:796. doi: 10.3390/app7080796

14. Flourat AL, Combes J, Bailly-Maitre-Grand C, Magnien K, Haudrechy A, Renault JH, et al. Accessing p-hydroxycinnamic acids: chemical synthesis, biomass recovery, or engineered microbial production? ChemSusChem. (2021) 14:118-29. doi: 10.1002/cssc.202002141

15. Sun Y, Wang W. Ultrasonic extraction of ferulic acid from Ligusticum chuanxiong \$. (2008) 39:653-6. doi: 10.1016/j.jcice.2008.05.012

16. Pazo-cepeda MV, Aspromonte SG, Alonso E. Food bioscience extraction of ferulic acid and feruloylated arabinoxylo-oligosaccharides from wheat bran using pressurized hot water. Food Biosci. (2021) 44:101374. doi: 10.1016/j.fbio.2021.101374

17. Cerullo G, Varriale S, Bozonnet S, Antonopoulou I, Christakopoulos P, Rova U, et al. (2019). Directed evolution of the type C feruloyl esterase from Fusarium oxysporum FoFaeC and molecular docking analysis of its improved variants. New Biotechnol. 51:14-20. doi: 10.1016/j.nbt.2019.01.008

18. Faulds CB, Williaon G. Release of ferulic acid from wheat bran by a ferulic acid esterase (FAE-III) from Aspergillus niger. Appl Microbiol Biotechnol. (1995) 43:1082-7. doi: 10.1007/BF00166929

19. Laszlo JA, Compton DL, Li XL. Feruloyl esterase hydrolysis and recovery of ferulic acid from jojoba meal. Ind Crops Prod. (2006) 23:46-53. doi: 10.1016/j.indcrop.2005.03.005

20. Topakas E, Vafiadi C, Christakopoulos P. Microbial production, characterization and applications of feruloyl esterases. Process Biochem. (2007) 42, 497-509. doi: 10.1016/j.procbio.2007.01.007

21. Juhnevica-Radenkova K, Kviesis J, Moreno DA, Seglina D, Vallejo F, Valdovska A, et al. Highly-efficient release of ferulic acid from agro-industrial by-products via enzymatic hydrolysis with cellulose-degrading enzymes: Part I-the superiority of hydrolytic enzymes versus conventional hydrolysis. Foods. (2021). 10:782. doi: 10.3390/foods10040782
22. Salgado JM, Max B, Rodríguez-Solana R, Domínguez JM. Purification of ferulic acid solubilized from agroindustrial wastes and further conversion into 4-vinyl guaiacol by Streptomyces setonii using solid state fermentation. Ind Crops Prod. (2012) 39:52-61. doi: 10.1016/j.indcrop.2012.02.014

23. Sibhatu HK, Anuradha Jabasingh S, Yimam A, Ahmed S. Ferulic acid production from brewery spent grains, an agro-industrial waste. Lwt. (2021) 135:110009. doi: 10.1016/j.lwt.2020.110009

24. Ou S, Luo Y, Xue F, Huang C, Zhang N, Liu Z. Seperation and purification of ferulic acid in alkaline-hydrolysate from sugarcane bagasse by activated charcoal adsorption/anion macroporous resin exchange chromatography. $J$ Food Eng. (2007) 78:1298-304. doi: 10.1016/j.jfoodeng.2005. 12.037

25. Dupoiron S, Lameloise ML, Pommet M, Bennaceur O, Lewandowski R, Allais F, Teixeira ARS, et al. A novel and integrative process: From enzymatic fractionation of wheat bran with a hemicellulasic cocktail to the recovery of ferulic acid by weak anion exchange resin. Indus Crops Prod. (2017) 105:148-55. doi: 10.1016/j.indcrop.2017.05.004

26. Dupoiron S, Lameloise ML, Bedu M, Lewandowski R, Fargues C, Allais F, et al. Recovering ferulic acid from wheat bran enzymatic hydrolysate by a novel and non-thermal process associating weak anionexchange and electrodialysis. Separat Purif Technol. (2018) 200:75-83. doi: 10.1016/j.seppur.2018.02.031

27. Gopalan N, Nampoothiri KM. Biorefining of wheat bran for the purification of ferulic acid. Biocatal Agric Biotechnol. (2018) 15:304-10. doi: 10.1016/j.bcab.2018.07.004

28. Ideia P, Sousa-ferreira I, Castilho PC. A novel and simpler alkaline hydrolysis brewer's spent grain and its (partial) purification. Foods. (2020) 9:600. doi: $10.3390 /$ foods 9050600

29. Sharma A, Sharma A, Singh J, Sharma P, Tomar GS, Singh S, et al. A biorefinery approach for the production of ferulic acid from agroresidues through ferulic acid esterase of lactic acid bacteria. Biotechnology. (2020) 10:1-10. doi: 10.1007/s13205-020-02360-9

30. Simon V, Thuret A, Candy L, Bassil S, Duthen S, Raynaud C, et al. Recovery of hydroxycinnamic acids from renewable resources by adsorption on zeolites. Chem Eng J. (2015) 280:748-54. doi: 10.1016/j.cej.2015.06.009

31. Qu Q, Tang W, Tang B, Zhu T. Highly selective purification of ferulic acid from wheat bran using deep eutectic solvents modified magnetic nanoparticles. Separat Sci Technol. (2017) 52:1022-30. doi: 10.1080/01496395.2017.1281299

32. Buranov AU, Mazza G. Extraction and purification of ferulic acid from flax shives, wheat and corn bran by alkaline hydrolysis and pressurised solvents. Food Chem. (2009) 115:1542-8. doi: 10.1016/j.foodchem.2009.01.059

33. Couteau D, Mathaly P. Purification of ferulic acid by adsorption after enzymic release from a sugar-beet pulp extract. Ind Crops Prod. (1997) 6:237-52. doi: 10.1016/S0926-6690(97)00014-9

34. Domingos JMB, Teixeira ARS, Dupoiron S, Allais F, Lameloise ML. Simultaneous recovery of ferulic acid and sugars from wheat bran enzymatic hydrolysate by diananofiltration. Separat Purif Technol. (2020) 242:116755. doi: 10.1016/j.seppur.2020.116755

35. Valério R, Crespo JG, Galinha CF, Brazinha C. Effect of ultrafiltration operating conditions for separation of ferulic acid from arabinoxylans in corn fibre alkaline extract. Sustainability. (2021) 13:1-16. doi: 10.3390/su13094682

36. Zhao S, Yao S, Ou S, Lin J, Wang Y, Peng X, et al. Preparation of ferulic acid from corn bran: Its improved extraction and purification by membrane separation. Food Bioprod Process. (2014) 92:309-13. doi: 10.1016/j.fbp.2013.09.004

37. Liu Z, Wang J, Shen P, Wang C, Shen Y. Microwave-assisted extraction and high-speed counter-current chromatography purification of ferulic acid from Radix Angelicae sinensis. Separat Purif Technol. (2006) 52:18-21. doi: 10.1016/j.seppur.2006.03.009

38. Tang Y, Hao J, Fan C, Cao X. Preparative separation of highpurity trans- and cis-ferulic acid from wheat bran by $\mathrm{pH}$-zone-refining counter-current chromatography. J Chromatogr A. (2021) 1636:461772. doi: 10.1016/j.chroma.2020.461772

39. Ren N, Wang C, Zhao Z, Liang Y, Wei W, Qin G. Recovery of ferulic acid from corn bran by adsorption on mesoporous carbon. J Food Process Eng. (2021) 44:1-8. doi: 10.1111/jfpe.13817 
40. Zhao Z, Egashira Y, Sanada H. Ferulic acid is quickly absorbed from rat stomach as the free form and then conjugated mainly in liver. J Nutr. (2004) 134:3083-8. doi: 10.1093/jn/134.11.3083

41. Wolffram S, Weber T, Grenacher B, Scharrer E. A $\mathrm{Na}(+)$-dependent mechanism is involved in mucosal uptake of cinnamic acid across the jejunal brush border in rats. J Nutr. (1995) 125:1300-8. doi: 10.1093/jn/125.5.1300

42. Spencer JPE, Chowrimootoo G, Choudhury R, Debnam ES, Srai SK, Rice-Evans C. The small intestine can both absorb and glucuronidate luminal flavonoids. FEBS Lett. (1999) 458:224-30. doi: 10.1016/S0014-5793(99)01160-6

43. Poquet L, Clifford MN, Williamson G. Transport and metabolism of ferulic acid through the colonic epithelium. Drug Metab Dispos. (2008) 36:190-7. doi: 10.1124/dmd.107.017558

44. Zhao Z, Egashira Y, Sanada H. Digestion and absorption of ferulic acid sugar esters in rat gastrointestinal tract. J Agric Food Chem. (2003) 51:5534-9. doi: $10.1021 /$ jf034455u

45. Zhao Z, Egashira Y, Sanada H. Ferulic acid sugar esters are recovered in rat plasma and urine mainly as the sulfoglucuronide of ferulic acid. J Nutr. (2003) 133:1355-61. doi: 10.1093/jn/133.5.1355

46. Tsuji A, Tamai I. Carrier-mediated intestinal transport of drugs. Pharm Res. (1996) 13:963-77. doi: 10.1023/A:1016086003070

47. Zhao Z, Moghadasian MH. Chemistry, natural sources, dietary intake and pharmacokinetic properties of ferulic acid: a review. Food Chem. (2008) 109:691-702. doi: 10.1016/j.foodchem.2008.02.039

48. Itagaki S, Kobayashi Y, Otsuka Y, Kubo S, Kobayashi M, Hirano T, et al. Food-drug interaction between ferrulic acid and nateglinide involving the fluorescein/H+ cotransport system. J Agric Food Chem. (2005) 53:2499-502. doi: 10.1021/jf047990i

49. Konishi Y, Shimizu M. Transepithelial transport of ferulic acid by monocarboxylic acid transporter in Caco-2 cell monolayers. (2003) 67:85662. doi: 10.1271/bbb.67.856

50. Watanabe H, Yashiro T, Tohjo Y, Konishi Y. Non-involvement of the human monocarboxylic acid transporter 1 (MCT1) in the transport of phenolic acid. (2006) 70:1928-33. doi: 10.1271/bbb.60093

51. Konishi Y, Zhao Z, Shimizu M. Phenolic acids are absorbed from the rat stomach with different absorption rates. J Agric Food Chem. (2006). 54:7539-7543. doi: 10.1021/jf061554+

52. Rondini L, Peyrat-Maillard MN, Marsset-Baglieri A, Berset C. Sulfated ferulic acid is the main in vivo metabolite found after short-term ingestion of free ferulic acid in rats. J Agric Food Chem. (2002) 50:3037-41. doi: 10.1021/jf011295i

53. Booth AN, Emerson OH, Jones FT, Deeds F. Urinary metabolites of caffeic and chlorogenic acids. J Biol Chem. (1957) 229:51-9. doi: 10.1016/S0021-9258(18)70592-9

54. Chesson A, Provan GJ, Russel WR, Scobbie L, Richardson AJ, Stewart C. Review Hydroxycinnamic acids in the digestive tract of livestock and humans. J Sci Food Agric. (1999) 378:373-8. doi: 10.1002/(SICI)10970010(19990301)79:3<373::AID-JSFA257>3.0.CO;2-6

55. Scheline RR. Metabolism of phenolic acids by the rat intestinal microflora the metabolism of several phenolic acids by intestinal contents has been reported by BOOTH. (1968) 26:189-205.

56. Kern SM, Bennet RN, Needs PW, Mellon FA, Kroon PA, Garcia-Conesa M-T. Characterization of metabolites of hydroxycinnamates in the in vitro model of human small intestinal epithelium Caco-2 cells. J Agric Food Chem. (2003). 7884-7891. doi: 10.1021/jf030470n

57. Xiao X-F, Gao M-Y, Zhu Q-H, Fan Mo H-LL, Gao S. Studies on pharmacokinetics and metabolism of ferulic acid. Asian J Pharmacodyn Pharmacokin. (2009) 9:135-43.

58. Li Y, Liu C, Zhang Y, Mi S, Wang N. Pharmacokinetics of ferulic acid and potential interactions with Honghua and clopidogrel in rats. $J$ Ethnopharmacol. (2011) 137:562-7. doi: 10.1016/j.jep.2011.06.011

59. de Paiva LB, Goldbeck R, dos Santos WD, Squina FM. Ferulic acid and derivatives: Molecules with potential application in the pharmaceutical field. Braz J Pharm Sci. (2013) 49:395-411. doi: 10.1590/S1984-82502013000300002

60. Chang MX, Xu LY, Tao JS, Feng Y. Metabolism and pharmacokinetics of ferulic acid in rats. China J Chin Mater Med. (1993) 18:300-19.
61. Kang J, Liu Y, Xie M, Li S, Jiang M, Wang Y. Interactions of human serum albumin with chlorogenic acid and ferulic acid. Biochim Biophys Acta. (2004) 1674:205-14. doi: 10.1016/j.bbagen.2004.06.021

62. Bourne LC, Rice-Evans C. Bioavailability of ferulic acid. Biochem Biophys Res Commun. (1998) 253:222-7. doi: 10.1006/bbrc.1998.9681

63. Choudhury R, Srai SK, Debnam E, Rice-Evans CA. Urinary excretion of hydroxycinnamates and flavonoids after oral and intravenous administration. Free Rad Biol Med. (1999) 27:278-86. doi: 10.1016/S0891-5849(99)00054-4

64. Jung H-JG, Fahey Jr GC. Effects of phenolic monomers on rat performance and metabolism. J Nutr. (1983) 113:546-556. doi: 10.1093/jn/113.3.546

65. Adam A, Crespy V, Levrat-Verny MA, Leenhardt F, Leuillet M, Demigné C, et al. (2002). The bioavailability of ferulic acid is governed primarily by the food matrix rather than its metabolism in intestine and liver in rats. J Nutr. 132:1962-8. doi: 10.1093/jn/132.7.1962

66. Panwar R, Raghuwanshi N, Srivastava AK, Sharma AK, Pruthi V. In-vivo sustained release of nanoencapsulated ferulic acid and its impact in induced diabetes. Mater Sci Eng C. (2018) 92:381-92. doi: 10.1016/j.msec.2018.06.055

67. Ou S, Kwok KC. Ferulic acid: pharmaceutical functions, preparation and applications in foods. J Sci Food Agric. (2004) 84:1261-9. doi: $10.1002 /$ jsfa. 1873

68. Zhao Z, Egashira Y, Sanada H. Phenolic antioxidants richly contained in corn bran are slightly bioavailable in rats. J Agric Food Chem. (2005) 53:5030-5. doi: 10.1021/jf050111n

69. Rondini L, Peyrat-Maillard MN, Marsset-Baglieri A, Fromentin G, Durand P. Bound ferulic acid from bran is more bioavailable than the free compound in rat. J Agric Food Chem. (2004) 52:4338-43. doi: 10.1021/jf0348323

70. Vardakou M, Nueno Palop C, Gasson M, Narbad A, Christakopoulos P. In vitro three-stage continuous fermentation of wheat arabinoxylan fractions and induction of hydrolase activity by the gut microflora. Int J Biol Macromol. (2007) 41:584-9. doi: 10.1016/j.ijbiomac.2007.07.017

71. Vardakou M, Palop CN, Christakopoulos P, Faulds CB, Gasson MA, Narbad A. Evaluation of the prebiotic properties of wheat arabinoxylan fractions and induction of hydrolase activity in gut microflora. Int J Food Microbiol. (2008) 123:166-70. doi: 10.1016/j.ijfoodmicro.2007.11.007

72. Ma Z, Hong Q, Wang Y, Liang Q, Tan H, Xiao C, et al. Ferulic acid induces heme oxygenase-1 via activation of ERK and Nrf2. Drug Discov Therap. (2011) 5:299-305. doi: 10.5582/ddt.2011.v5.6.299

73. Wang X, Stavchansky S, Kerwin SM, Bowman PD. Structure-activity relationships in the cytoprotective effect of caffeic acid phenethyl ester (CAPE) and fluorinated derivatives: Effects on heme oxygenase-1 induction and antioxidant activities. Eur J Pharmacol. (2010) 635:16-22. doi: 10.1016/j.ejphar.2010.02.034

74. Zhu Y, Shao Y, Qu X, Guo J, Yang J, Zhou Z, et al. Sodium ferulate protects against influenza virus infection by activation of the TLR7/9-MyD88IRF7 signaling pathway and inhibition of the NF- $\mathrm{B}$ signaling pathway. Biochem Biophys Res Commun. (2019) 512:793-8. doi: 10.1016/j.bbrc.2019. 03.113

75. Sakai S, Kawamata H, Kogure T, Mantani N, Terasawa K, Umatake M, et al. Inhibitory effect of ferulic acid and isoferulic acid on the production of macrophage inflammatory protein-2 in response to respiratory syncytial virus infection in RAW264. Cells. (1999) 175:173-5.

76. Hirabayashi T, Ochiai H, Sakai S, Nakajima K, Terasawa K. Inhibitory effect of ferulic acid and isoferulic acid on murine interleukin- 8 production in response to influenza virus infections in vitro and in vivo. Planta Medica. (1995) 61:221-6. doi: 10.1055/s-2006-958060

77. McCarty MF, DiNicolantonio JJ. Nutraceuticals have potential for boosting the type 1 interferon response to RNA viruses including influenza and coronavirus. Prog Cardiovasc Dis. (2020) 63:383-5. doi: 10.1016/j.pcad.2020.02.007

78. Hariono M, Abdullah N, Damodaran KV, Kamarulzaman EE, Mohamed N, Hassan SS, et al. Potential new H1N1 neuraminidase inhibitors from ferulic acid and vanillin: molecular modelling, synthesis and in vitro assay. Sci Rep. (2016) 6:1-10. doi: 10.1038/srep38692

79. Salman S, Shah FH, Idrees J, Idrees F, Velagala S, Ali J, et al. Virtual screening of immunomodulatory medicinal compounds as promising anti-SARS-CoV2 inhibitors. Future Virol. (2020) 15:267-75. doi: 10.2217/fvl-2020-0079 
80. Bhowmik D, Nandi R, Jagadeesan R, Kumar N, Prakash A, Kumar D. Identification of potential inhibitors against SARS-CoV-2 by targeting proteins responsible for envelope formation and virion assembly using docking based virtual screening, and pharmacokinetics approaches. Infect Genet Evol. (2020) 84:104451. doi: 10.1016/j.meegid.2020.104451

81. Ma X, Guo Z, Zhang Z, Li X, Wang X, Liu Y, et al. Ferulic acid isolated from propolis inhibits porcine parvovirus replication potentially through Bid-mediate apoptosis. Int Immunopharmacol. (2020) 83:106379. doi: 10.1016/j.intimp.2020.106379

82. Dimitrova Z, Dimov B, Manolova N, Pancheva S, Ilieva D, Shishkov S. Antiherpes effect of Melissa officinalis L. extracts. Acta Microbiol Bulg. (1993) 29:65-72.

83. Chiang LC, Chiang W, Chang MY, Ng LT, Lin CC. Antiviral activity of Plantago major extracts and related compounds in vitro. Antiviral Res. (2002) 55:53-62. doi: 10.1016/S0166-3542(02)00007-4

84. Aref HL, Gaaliche B, Fekih A, Mars M, Aouni M, Chaumon JP, et al. (2011). In vitro cytotoxic and antiviral activities of Ficus carica latex extracts. Nat Prod Res. (2011) 25:310-19. doi: 10.1080/14786419.2010.528758

85. Wang CY, Huang SC, Zhang Y, Lai ZR, Kung SH, Chang YS, et al. Antiviral ability of Kalanchoe gracilis leaf extract against enterovirus 71 and coxsackievirus A16. Evid Based Complem Altern Med. (2012) 2012:503165. doi: 10.1155/2012/503165

86. John KMM, Ayyanar M, Jeeva S, Suresh M, Enkhtaivan G, Kim DH. Metabolic variations, antioxidant potential, and antiviral activity of different extracts of Eugenia singampattiana (an Endangered Medicinal Plant Used by Kani Tribals, Tamil Nadu, India). Leaf BioMed Res Int. (2014) 2014:726145. doi: 10.1155/2014/726145

87. Bankova V, Galabov AS, Antonova D, Vilhelmova N, Di Perri B. Chemical composition of Propolis Extract ACF $\AA$ and activity against herpes simplex virus. Phytomedicine. (2014) 21:1432-8. doi: 10.1016/j.phymed.2014.04.026

88. Kwon MJ, Shin HM, Perumalsamy H, Wang X, Ahn YJ. Antiviral effects and possible mechanisms of action of constituents from Brazilian propolis and related compounds. J Apic Res. (2020) 59:413-25. doi: 10.1080/00218839.2019.1695715

89. Enkhtaivan G, Maria John KM, Ayyanar M, Sekar T, Jin KJ, Kim DH. Anti-influenza (H1N1) potential of leaf and stem bark extracts of selected medicinal plants of South India. Saudi J Biol Sci. (2015) 22:532-8. doi: 10.1016/j.sjbs.2015.01.011

90. Joshi SS, Dice L, D'Souza DH. Aqueous extracts of Hibiscus sabdariffa calyces decrease hepatitis a virus and human norovirus surrogate titers. Food Environ Virol. (2015) 7:366-73. doi: 10.1007/s12560-015-9209-1

91. Abdelkhalek A, Salem MZM, Hafez E, Behiry SI. The antiviral properties of egyptian Haplophyllum tuberculatum extract. Biology. (2020) 9:1-17. doi: 10.3390/biology9090248

92. Di Sotto A, Checconi P, Celestino I, Locatelli M, Carissimi S, De Angelis $\mathrm{M}$, et al. (2018). Antiviral and antioxidant activity of a hydroalcoholic extract from humulus lupulus L. Oxid Med Cell Long. (2018) 2018:5919237. doi: 10.1155/2018/5919237

93. Landry KB, Azam S, Rehman S, Tariq S, Iqbal B, Abbas M, et al. Phytochemical analysis of Berberis lyceum methanolic extract and its antiviral activity through the restoration of MAPK signaling pathway modulated by HCV NS5A. Asian Pac J Trop Biomed. (2021) 11:132-40. doi: 10.4103/2221-1691.306133

94. Silva-Beltrán NP, Ruiz-Cruz S, Chaidez C, Ornelas-Paz JDJ, López-Mata MA, Márquez-Riós E, et al. Chemical constitution and effect of extracts of tomato plants byproducts on the enteric viral surrogates. Int J Environ Health Res. (2015) 25:299-311. doi: 10.1080/09603123.2014.938030

95. Silva-Beltrán NP, Chaidez-Quiroz C, López-Cuevas O, Ruiz-Cruz S, López-Mata MA, Del-Toro-Sánchez CL, Marquez-Rios E, et al. Phenolic compounds of potato peel extracts: their antioxidant activity and protection against human enteric viruses. J Microbiol Biotechnol. (2017) 27:234-41. doi: 10.4014/jmb.1606.06007

96. Abdelkhalek A, Salem MZM, Kordy AM, Salem AZM, Behiry SI. Antiviral, antifungal, and insecticidal activities of Eucalyptus bark extract: HPLC analysis of polyphenolic compounds. Microb Pathog. (2020) 147:104383. doi: 10.1016/j.micpath.2020.104383

97. Akihisa T, Ogihara J, Kato J, Yasukawa K, Ukiya M, Yamanouchi $\mathrm{S}$, et al. Inhibitory effects of triterpenoids and sterols on human immunodeficiency virus-1 reverse transcriptase. Lipids. (2001) 36:507-12. doi: 10.1007/s11745-001-0750-4

98. De Jesus Silva Júnior A, De Campos-Buzzi F, Romanos MTV, Wagner TM, De Paula Costa Guimarães AF, Filho VC, et al. Chemical composition and antinociceptive, anti-inflammatory and antiviral activities of Gallesia gorazema (Phytolaccaceae), a potential candidate for novel anti-herpetic phytomedicines. J Ethnopharmacol. (2013) 150:595-600. doi: 10.1016/j.jep.2013.09.005

99. Sekizawa H, Ikuta K, Ohnishi-Kameyama M, Nishiyama K, Suzutani T. Identification of the components in a vaccinium oldhamii extract showing inhibitory activity against influenza virus adsorption. Foods. (2019) 8:8050172. doi: 10.3390/foods 8050172

100. Mazzanti G, Battinelli L, Pompeo C, Serrilli AM, Rossi R, Sauzullo I, et al. Inhibitory activity of Melissa officinalis L. extract on Herpes simplex virus type 2 replication. Nat Prod Res. (2008) 22:1433-40. doi: 10.1080/14786410802075939

101. Moradi MT, Karimi A, Alidadi S, Hashemi L. In vitro anti-adenovirus activity, antioxidant potential and total phenolic compounds of Melissa officinalis L. (lemon balm) extract. Int J Pharmacogn Phytochem Res. (2016) 8:1471-7.

102. Kim H-Y, Shin H-S, Park H, Kim Y-C, Yun YG, Park S, et al. In vitro inhibition of coronavirus replications by the traditionally used medicinal herbal extracts, Cimicifuga rhizoma, Meliae cortex, Coptidis rhizoma, and Phellodendron cortex. J Clin Virol. (2008) 41:122-8. doi: 10.1016/j.jcv.2007.10.011

103. Jang JY, Lee JH, Kang BW, Chung KT, Chol YH, Choi BT. Dichloromethane fraction of Cimicifuga heracleifolia decreases the level of melanin synthesis by activating the ERK or AKT signaling pathway in B16F10 cells. Exp Dermatol. (2009) 18:232-7. doi: 10.1111/j.1600-0625.2008.00794.x

104. Ichimura T, Otake T, Mori H, Maruyama S. HIV-1 protease inhibition and anti-HIV effect of natural and synthetic water-soluble lignin-like substances. Biosci Biotechnol Biochem. (1999) 63:2202-4. doi: 10.1271/bbb.63.2202

105. Spasova M, Philipov S, Nikolaeva-Glomb L, Galabov AS, Milkova T. Cinnamoyl- and hydroxycinnamoyl amides of glaucine and their antioxidative and antiviral activities. Bioorg Med Chem. (2008) 16:7457-61. doi: 10.1016/j.bmc.2008.06.010

106. Huang GY, Cui C, Wang ZP, Li YQ, Xiong LX, Wang LZ, et al. Synthesis and characteristics of (Hydrogenated) ferulic acid derivatives as potential antiviral agents with insecticidal activity. Chem Cent J. (2013) 7:1-12. doi: 10.1186/1752-153X-7-33

107. Cui C, Wang ZP, Du XJ, Wang LZ, Yu SJ, Liu XH, et al. Synthesis and antiviral activity of hydrogenated ferulic acid derivatives. J Chem. (2013) 2013:269434. doi: 10.1155/2013/269434

108. Lan X, Xie D, Yin L, Wang Z, Chen J, Zhang A, et al. Novel $\alpha, \beta$ unsaturated amide derivatives bearing $\alpha$-amino phosphonate moiety as potential antiviral agents. Bioorg Med Chem Lett. (2017) 27:4270-3. doi: 10.1016/j.bmcl.2017.08.048

109. Wang Z, Xie D, Gan X, Zeng S, Zhang A, Yin L, et al. Synthesis, antiviral activity, and molecular docking study of trans-ferulic acid derivatives containing acylhydrazone moiety. Bioorg Med Chem Lett. (2017) 27:4096100. doi: 10.1016/j.bmcl.2017.07.038

110. Ren X, Li X, Yin L, Jiang D, Hu D. Design, synthesis, antiviral bioactivity, and mechanism of the ferulic acid ester-containing sulfonamide moiety. ACS Omega. (2020) 5:19721-6. doi: 10.1021/acsomega.0c02421

111. Tang X, Zhang C, Chen M, Xue Y, Liu T, Xue W. Synthesis and antiviral activity of novel myricetin derivatives containing ferulic acid amide scaffolds. New J Chem. (2020) 44:2374-9. doi: 10.1039/c9nj05867b

112. Wu Z, Zhang J, Chen J, Pan J, Zhao L, Liu D, et al. Design, synthesis, antiviral bioactivity and three-dimensional quantitative structure-activity relationship study of novel ferulic acid ester derivatives containing quinazoline moiety. Pest Manag Sci. (2017) 73:2079-89. doi: 10.1002/ps. 4579

113. Gan X, Hu D, Wang Y, Yu L, Song B. Novel trans-ferulic acid derivatives containing a chalcone moiety as potential activator for plant resistance induction. J Agric Food Chem. (2017) 65:4367-77. doi: 10.1021/acs.jafc.7b00958

114. Wang Y, He F, Wu S, Luo Y, Wu R, Hu D, et al. Design, synthesis, antiTMV activity, and preliminary mechanism of cinnamic acid derivatives 
containing dithioacetal moiety. Pesticide Biochem Physiol. (2020) 164:11521. doi: 10.1016/j.pestbp.2020.01.002

115. Li N-G, Shi Z-H, Tang Y-P, Li B-Q, Duan J-A. Highly efficient esterification of ferulic acid under microwave irradiation. Molecules. (2009) 14:2118. doi: 10.3390/molecules14062118

116. Lee GS, Widjaja A, Ju YH. Enzymatic synthesis of cinnamic acid derivatives. Biotechnol Lett. (2006) 28:581-5. doi: 10.1007/s10529-006-0019-2

117. Katayama S, Ohno F, Yamauchi Y, Kato M, Makabe H, Nakamura S. Enzymatic synthesis of novel phenol acid rutinosides using rutinase and their antiviral activity in vitro. J Agric Food Chem. (2013) 61:9617-22. doi: 10.1021/jf4021703

118. Gamayurova VS, Zinov'eva ME, Shnaider KL, Davletshina GA. Lipases in esterification reactions: a review. Catal. Indus. (2021) 13:58-72. doi: 10.1134/S2070050421010025

119. Kumar A, Kanwar SS. Synthesis of ethyl ferulate in organic medium using celite-immobilized lipase. Bioresour Technol. (2011) 102:2162-7. doi: 10.1016/j.biortech.2010.10.027

120. Sabally K, Karboune S, St-Louis R, Kermasha S. Lipase-catalyzed transesterification of trilinolein or trilinolenin with selected phenolic acids. $J$ Am Oil Chem Soc. (2006) 83:101-7. doi: 10.1007/s11746-006-1181-3

121. Matsuo T, Kobayashi T, Kimura Y, Hosoda A, Taniguchi H, Adachi S. Continuous synthesis of glyceryl ferulate using immobilized Candida antarctica lipase. J Oleo Sci. (2008) 57:375-80. doi: 10.5650/jos. 57.375

122. Zheng Y, Wu XM, Branford-White C, Quan J, Zhu LM. Dual response surface-optimized process for feruloylated diacylglycerols by selective lipasecatalyzed transesterification in solvent free system. Bioresour Technol. (2009) 100:2896-901. doi: 10.1016/j.biortech.2009.01.042

123. Ishihara K, Katsube Y, Kumazawa N, Kuratani M, Masuoka N, Nakajima N. Enzymatic preparation of arbutin derivatives: Lipase-catalyzed direct acylation without the need of vinyl ester as an acyl donor. J Biosci Bioeng. (2010) 109:554-6. doi: 10.1016/j.jbiosc.2009.11.009

124. Xin J, Chen L, Zhang Y, Wen R, Zhao D, Xia C. Lipase-catalyzed synthesis of $\alpha$-tocopheryl ferulate. Food Biotechnol. (2011) 25:43-57. doi: 10.1080/08905436.2011.547116

125. Tan Z, Shahidi F. Chemoenzymatic synthesis of phytosteryl ferulates and evaluation of their antioxidant activity. J Agric Food Chem. (2011) 59:1237583. doi: 10.1021/jf2034237

126. Kumar V, Jahan F, Mahajan RV, Saxena RK. Efficient regioselective acylation of quercetin using Rhizopus oryzae lipase and its potential as antioxidant. Bioresour Technol. (2016) 218:1246-8. doi: 10.1016/j.biortech.2016.06.057

127. Schär A, Nyström L. Enzymatic synthesis of steryl ferulates. European Journal of Lipid Science and Technology. (2016) 118:1557-65. doi: 10.1002/ejlt.201500586

128. Bondhon TA, Mahamud R, Al Jannat K, Hasan A, Jahan R, Rahmatullah M. In silico binding studies with b-sitosterol and some of its fatty acid esters to 3C-like protease of SARS-CoV-2. J Med Plants Stud. (2020). 8:86-90. doi: 10.22271/plants.2020.v8.i5b.1198

129. Katsimpouras C, Antonopoulou I, Christakopoulos P, Topakas E. Role and applications of feruloyl esterases in biomass bioconversion. In: Microbial Enzymes in Bioconversions of Biomass, ed V. K. Gupta. Springer International Publishing (2016), pp. 79-123.

130. Oliveira DM, Mota TR, Oliva B, Segato F, Marchiosi R, Ferrarese-Filho $\mathrm{O}$, et al. Feruloyl esterases: Biocatalysts to overcome biomass recalcitrance and for the production of bioactive compounds. Biores Technol. (2019) 278:408-23. doi: 10.1016/j.biortech.2019.01.064

131. Faulds CB, Pérez-Boada M, Martínez ÁT. Influence of organic co-solvents on the activity and substrate specificity of feruloyl esterases. Bioresource Technol. (2011) 102:4962-7. doi: 10.1016/j.biortech.2011.01.088

132. Antonopoulou I, Iancu L, Jütten P, Piechot A, Rova U, Christakopoulos P. Screening of novel feruloyl esterases from Talaromyces wortmannii for the development of efficient and sustainable syntheses of feruloyl derivatives. Enzyme Microb Technol. (2019). 120:124-35. doi: 10.1016/j.enzmictec.2018.08.007

133. Couto J, St-Louis R, Karboune S. Optimization of feruloyl esterase-catalyzed synthesis of feruloylated oligosaccharides by response surface methodology. J Mol Catal B Enzym. (2011) 73:53-62. doi: 10.1016/j.molcatb.2011.07.016

134. Hüttner S, Zezzi Do Valle Gomes M, Iancu L, Palmqvist A, Olsson L. Immobilisation on mesoporous silica and solvent rinsing improve the transesterification abilities of feruloyl esterases from Myceliophthora thermophila. Bioresour Technol. (2017) 239:57-65. doi: 10.1016/j.biortech.2017.04.106

135. Topakas E, Stamatis H, Biely P, Kekos D, Macris BJ, Christakopoulos P. Purification and characterization of a feruloyl esterase from Fusarium oxysporum catalyzing esterification of phenolic acids in ternary water-organic solvent mixtures. J Biotechnol. (2003) 102:33-44. doi: 10.1016/S0168-1656(02)00363-2

136. Varriale S, Cerullo G, Antonopoulou I, Christakopoulos P, Rova U, Tron T, et al. Evolution of the feruloyl esterase MtFaela from Myceliophthora thermophila towards improved catalysts for antioxidants synthesis. Appl Microbiol Biotechnol. (2018) 102:5185-96. doi: 10.1007/s00253-018-8995-4

137. Zerva A, Antonopoulou I, Enman J, Iancu L, Jütten P, Rova U, et al. Optimization of transesterification reactions with CLEA-Immobilized feruloyl esterases from thermothelomyces thermophila and talaromyces wortmannii. Molecules. (2018) 23:1-16. doi: 10.3390/molecules23092403

138. Adelakun OE, Kudanga T, Parker A, Green IR, Le Roes-Hill M, Burton SG. Laccase-catalyzed dimerization of ferulic acid amplifies antioxidant activity. J Mol Catal B Enzym. (2012) 74:29-35. doi: 10.1016/j.molcatb.2011. 08.010

Conflict of Interest: The authors declare that the research was conducted in the absence of any commercial or financial relationships that could be construed as a potential conflict of interest.

Publisher's Note: All claims expressed in this article are solely those of the authors and do not necessarily represent those of their affiliated organizations, or those of the publisher, the editors and the reviewers. Any product that may be evaluated in this article, or claim that may be made by its manufacturer, is not guaranteed or endorsed by the publisher.

Copyright (C) 2022 Antonopoulou, Sapountzaki, Rova and Christakopoulos. This is an open-access article distributed under the terms of the Creative Commons Attribution License (CC BY). The use, distribution or reproduction in other forums is permitted, provided the original author(s) and the copyright owner(s) are credited and that the original publication in this journal is cited, in accordance with accepted academic practice. No use, distribution or reproduction is permitted which does not comply with these terms. 